\title{
Molecular mechanism of ultrasound interaction with a blood brain barrier model
}

\author{
Viet Hoang Man ${ }^{1}$, Mai Suan $\mathrm{Li}^{2,3}$, Philippe Derreumaux ${ }^{4,5}$, Junmei \\ Wang $^{1}$, Toan T. Nguyen ${ }^{6}$, S. Nangia ${ }^{7}$, Phuong H. Nguyen ${ }^{4,5 *}$ \\ 1, Department of Pharmaceutical Sciences, University of Pittsburgh, USA \\ ${ }^{2}$ Institute of Physics, Polish Academy of Sciences, Al. Lotnikow 32/46, 02-668 Warsaw, Poland \\ ${ }^{3}$ Institute for Computational Science and Technology, SBI Building, \\ Quang Trung Software City, Tan Chanh Hiep Ward, District 12, Ho Chi Minh City, Vietnam \\ ${ }^{4}$ CNRS, Universite de Paris, UPR9080, Laboratoire de Biochimie Théorique, Paris, France \\ ${ }^{5}$ Institut de Biologie Physico-Chimique Fondation Edmond de Rothschlid, PSL Research University, Paris, France \\ ${ }^{6}$ Key Laboratory for Multiscale Simulation of Complex Systems, \\ VNU University of Science, Vietnam National University, \\ 334 Nguyen Trai Street, Thanh Xuan, Hanoi, Vietnam \\ ${ }^{7}$ Department of Biomedical and Chemical Engineering, \\ Syracuse University, Syracuse, NY 13244, USA.
}

(Dated: June 25, 2020)

The brain is strictly protected by the blood brain barrier preventing the crossing of therapeutics to treat brain diseases. The high and low intensity focused ultrasound methods have been used to open temporarily the blood brain barrier, facilitating the transport of drugs. The methods are very promising because the opening is transient, localized and noninvasive. However, the molecular mechanism of the opening is unknown, and this limits the development and application of these methods. With this in mind, we carry out a molecular dynamics simulation study to understand the interaction of ultrasound with the cell membrane and the tight junction. Our minimal blood brain barrier model is composed of two lipid bilayers, mimicking two portions of neighbouring cells, connected together by a tight junction formed by a pair of two cis-dimers of the claudin- 5 protein. Using an experimental ultrasound frequency of $50 \mathrm{MHz}$, simulations show that at low intensities, ultrasound does not impact the structure of the cell membranes and tight junction, implying that the direct interaction of ultrasound with the blood brain barrier is not responsible for the experimentally observed opening. At high intensities, the ultrasound pulls the monolayers of individual cell membrane lipid bilayers apart, creating air compartments inside the bilayers. This reduces the free energy barrier for the translocation of drugs across the lipid bilayer and enhances drug permeability. At very high intensities, the two monolayers are largely separated, resulting in cell damage and implying that the blood brain barrier is primarily opened at the experimentally observed damaged areas.

\section{INTRODUCTION}

The blood brain barrier (BBB) acts as the permeability barrier between the blood capillary and the brain [1]. It is composed of endothelial cells connected together by junctional complexes consisting of tight junctions and adherens junctions. As a general rule, only small lipid soluble molecules with a molecular mass $\leq 500$ Da may cross the BBB. Otherwise, different molecules may gain access to the brain only via certain endogenous transport systems within the $\mathrm{BBB}[2,3]$. As a consequence, only about $5 \%$ of $\sim 7000$ known potential drugs for the brain treatment can cross the BBB[4].

As a remedy, several methods have been developed aimed at enhancing the permeability of drugs through the $\mathrm{BBB}\left[5_{-}^{-9]}\right.$. Among these methods, the use of focused ultrasound is very promising as it is truly transient, localized and noninvasive for temporarily opening the BBB[10-14]. The very first study was carried out in 1955 by Barnard et al. to study the BBB permeabil-

*Email: nguyen@ibpc.fr ity following high intensity focused ultrasound (HIFU) irradiation[15]. They showed that blood vessels were not altered morphologically by ultrasound. The following study by Bakay et al. showed that the BBB appeared to be altered, made easily permeable within the area damaged by HIFU[16]. Later, Patrick et al. noted that the $\mathrm{BBB}$ is also disrupted at the periphery of the HIFUinduced lesions[17]. Ballantine et al. used a high-power defocused ultrasonic beam and showed, for the first time, that it may be possible to select ultrasound parameters that allow for a BBB disruption without risk of producing lesions[18]. In the next step, Vykhodtseva et al. used a variety of short burst durations and pulse repetition frequencies of HIFU to visualise effects in rabbit brains, and observed sometimes BBB opening without apparent damage to the brain parenchyma[19]. However, the ultrasound parameters that could produce this effect consistently were not found. Finally, Mesiwala et al. reported results in the rat brain showing that HIFU sometimes produces BBB disruption without visible damage to the brain parenchyma. They suggested that opening of the tight junctions could be the mechanism of the BBB disruption. However, no tracer molecules were used in this study to prove this mechanism passage[11]. Taken to- 
gether, after many decades, the induced-HIFU molecular mechanism of BBB opening is still unclear.

One of the reasons that prevents further development of the HIFU method is probably due to the fact that a safer method was latter developed by Hynynen and colleagues in 2001. In this method, a low intensity focused ultrasound (LIFU) is used in combination with microbubbles to open the BBB. Microbubbles concentrate ultrasound energy to desirable areas, thus low intensity ultrasound is sufficient to open the BBB, and cell lesions could therefore be minimised[10]. Since then, a large number of studies have been carried both in in vitro and in vivo, focusing on different aspects. One of the important aspects is the fundamental interaction mechanisms of LIFU with microbubbles, and effects of bubble cavitation on the BBB[20]. Another aspect is the influence of ultrasound parameters such as pressure amplitude, frequency, burst length, pulse repetition frequency, sonication duration, and parameters of microbubbles such as dose, diameter and type, on the BBB opening mechanism[21-24]. Also, many works have focused on the quantification of the permeability of the disrupted brain tissues [25, 26], test the delivery of new drugs, evaluate and monitor the response of treatments[27-30]. Nevertheless, the exact molecular mechanism of the LIFU induced BBB opening is still unknown.

Compared to experiments, theoretical studies of the ultrasound induced BBB opening are still at their infancy. Several mathematical models have been developed, which are essentially composed of coupled partial differential equations and solved numerically[31-37]. These studies provide valuable information on the effect of bubbles cavitation and ultrasound on the vessel wall. However, relying on continuum mechanics, these models cannot capture detailed conformational changes of the BBB at the molecular level. At the molecular level, a number of simulations employing coarse-grained and atomistic molecular models have been carried out to study the effects of the bubble inertial cavitation and shock waves on the cell membranes. These studies have confirmed that inertial cavitation can induce the formation of membrane pores[38-47]. Recently, a molecular dynamics (MD) simulation study based on a coarse-grained model explored the possibility of opening a simple tight junction due to shock wave induced bubble collapse[48].

The molecular mechanisms of the BBB opening under HIFU and LIFU irradiations are very complex, because they results from interaction of ultrasound and bubble interaction with the cellular membranes and the tight junctions. To simulate these interactions, in principle we must have a bubble model, an ultrasound simulation method and a molecular BBB model. With this in mind, we have recently developed a bubble model and a focused ultrasound simulation method[49-51]. This allows us to study the molecular mechanism of the interaction between ultrasound with lipid cell membrane, in the presence of bubble[51] or without bubble[52]. For the molecular BBB model, Nangia and coworkers have recently obtained the structure of the claudin- 5 protein via homology modelling of the experimental structure of the claudin-15 protein. Their MD simulations have shown that claudin-5 monomers embedded in seven membranes with different lipid compositions readily form stable cisdimers, which subsequently form contiguous strands and high-order assemblies[53]. These cis-dimers are used to construct the trans interfaces, which are formed when claudin-5 proteins on adjacent cells interact head-on to form the tight junction assembly, by using docking methods in combination with symmetric refinement[54]. Fundamental pore features, such as diameter and length of the pore were characterized in details [55].

As a first attempt to provide more insights into the ultrasound induced BBB opening molecular mechanism due to HIFU or LIFU, in this work, based on the above mentioned achievements, we carry out MD simulations aimed at understanding the impact of ultrasound alone on the cell membranes and tight junction using the minimal BBB model. In particular, we wish to understand which part of the $\mathrm{BBB}$ model: membrane or tight junction resists more to the ultrasound. The impacts of the bubble cavitation, and the construction of more sophisticated BBB models will be carried out in next studies.

\section{METHODS}

\section{A. The minimal BBB model}

The family of claudin proteins forms tight junctions in endothelial and epithelial cells[56]. Among 27 known members of the claudin family, the claudin-5 is the key tight junction protein with an expression level that is much higher than other claudin proteins[57-59]. Thus, we use only claudin- 5 protein to construct the tight junction. Our minimal BBB model consists of two lipid bilayer membranes connected together by a paracellular pore formed by two claudin-5 cis-dimers. The molecular structure is shown in Fig.1 and briefly described here. First, the structure of the claudin-5 monomer is obtained via homology modelling of the experimental structure of the claudin-15 protein (PDB ID: 4P79). The claudin-5 protein consists of 218 residues, forming four transmembrane helix bundles (TM1-4), two extracellular loops (ECL1, ECL2) and an intracellular loop (ICL). The structure of a claudin-5 cis-dimer in the phosphatidylcholine (POPC) membrane was obtained from previous MD simulations (model B)[53]. In this structure, two claudin- 5 monomers are mediated by the interaction between leucine residues 83, 90 of TM2 of one monomer and residues 124,131 of TM3 of another monomer, and flanking aromatic $\pi-\pi$ interactions of Phe127 and Trp138 of two TM3. Then, a pair of two cis-dimers is used to construct the paracellular pore by using the docking methods in combination with symmetric refinement. This trans pair is stablized by hydrogen-bonds and salt bridges between ECL contacts. This minimal BBB model captures 

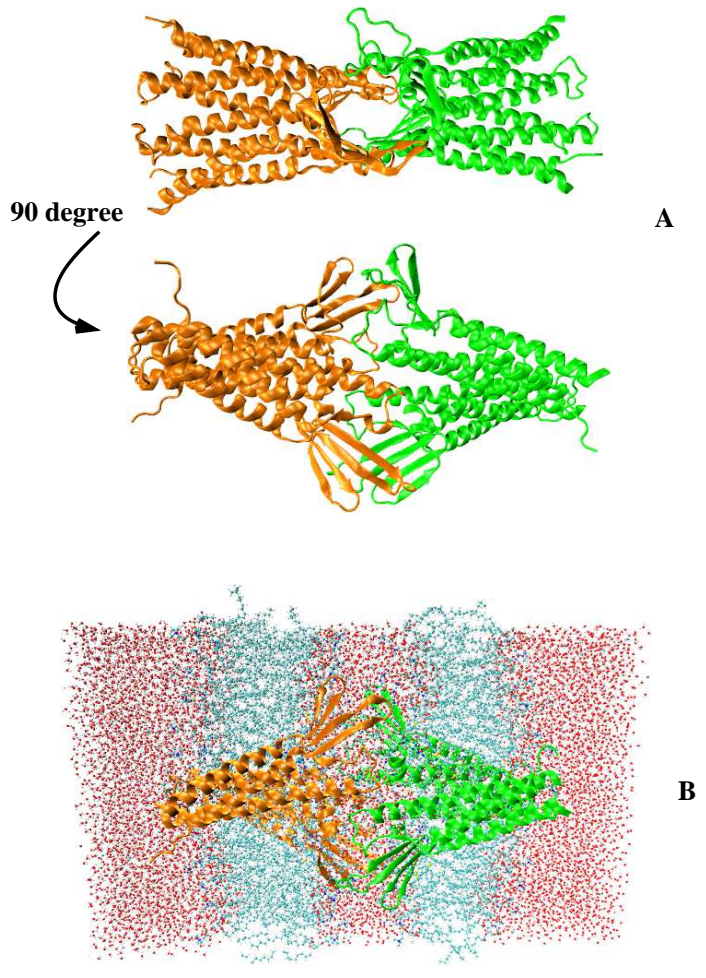

Figure 1: (A) The molecular structure, viewed from two angles, of two claudin- 5 cis-dimers (green and orange) forming a paracellular pore. (B) The molecular structure of our minimal BBB model where cis-dimers are embed into two lipid membranes of two neighbouring cells. The water is filled between and outside two lipid bilayers.

the fundamental interactions responsible for the $\mathrm{BBB}$ tight junction assembly[54, 55], and serves as a starting point for simulating effect of ultrasound on the BBB. We note that our BBB model is quite similar to that from Ref.[48], which is composed of a pair of two claudin-15 dimers at the TJ

We use the all-atom CHARMM36 force field[60] to model the protein and POPC lipid, and the TIP3P water model[61] to describe solvent. The whole model is solvated in a water box consisting of $\sim 180000$ waters. The initial dimensions of the unit cell are $\left(L_{x}, L_{y}, L_{z}\right)=$ $(10,10,18) \mathrm{nm}$. Starting from this structure, an equilibrium MD simulation is carried out for $100 \mathrm{~ns}$ in the NPT ensemble with the pressure $P_{0}=1 \mathrm{bar}$ and temperature $T=300 \mathrm{~K}$, employing the GROMACS simulation package[62]. The last structure is used as initial structure for ultrasound simulations.

\section{B. The ultrasound simulation method}

In a conventional simulation, the pressure of the system is maintained at a desired value $P_{0}$ by using a barostat. Currently, the Berendsen barostat is widely used as it is simple in both theory and implementation. Basically, it consists of an external bath so that the global pressure of the system is weakly coupled to this pressure bath by rescaling periodically the lengths of the system[63]

$$
\mathbf{r}_{i} \rightarrow \mu \mathbf{r}_{i} \quad(i=1 \cdots N) \text { and } L \rightarrow \mu L,
$$

where $N, \mathbf{r}_{i}$ and $L$ are, respectively, the number of atoms in the system, the coordinate of the $i$-th atom and the length of the system box. The scale factor is given by

$$
\mu=\left[1-\frac{\beta \Delta t}{\tau_{p}}\left(P(t)-P_{0}\right)\right]^{1 / 3},
$$

where $P(t), \beta, \Delta t$, and $\tau_{p}$ are the instantaneous pressure in the system, the isothermal compressibility, the integration time step and the temperature coupling constant, respectively. After scaling lengths through Eq. 1, the system volume becomes $V \rightarrow\left[1-\frac{\beta \Delta t}{\tau_{p}}\left(P(t)-P_{0}\right)\right] V$. This ways, the instantaneous pressure $P(t)$ always fluctuates around the desired value $P_{0}$.

In an ultrasound simulation, the instantaneous pressure of the system will be increased and decreased around the reference value $P_{0}$ following the compression and rarefaction of the sound wave. We take into account this effect of the ultrasound wave, which has the form

$$
p(t)=A \sin (2 \pi \omega t)
$$

by using a modified scale factor

$$
\mu=\left[1-\frac{\beta \Delta t}{\tau_{p}}\left(P(t)-P_{0}-A \sin (2 \pi \omega t)\right)\right]^{1 / 3},
$$

where $\omega$ and $A$ are the frequency and amplitude of the ultrasound. The lengths of the system are then scaled as usual (Eq.1). This guarantees that the pressure of the system is always equal to the pressure of the ultrasound.

The GROMACS simulation package[62] coupled to our code of ultrasound is used for all the simulations. In all simulations, the ultrasound with frequency $\omega=20 \mathrm{MHz}$ is used, and the amplitude is varied from 100 to 500 bar. The reference pressure, $P_{0}=1$ bar, the pressure coupling constant, $\tau_{p}=1 \mathrm{ps}$ and the isothermal compressibility $\beta=4.5 \times 10^{-5}$ bar $^{-1}$ are used. To ensure that the damage to the membrane is not due to heat generated by work done by ultrasound, we couple both membrane and water to the heat bath at $300 \mathrm{~K}$ employing the Berendsen coupling method[63] with a temperature coupling constant of 0.1 ps. The equations of motion are integrated using the leapfrog algorithm with a small time step of $2 \mathrm{fs}$. The electrostatic interactions are calculated using the particle mesh Ewald method and a cutoff of $1.4 \mathrm{~nm}$ [64]. A cutoff of $1.4 \mathrm{~nm}$ is used for the van der Waals interactions. The nonbonded pair lists are updated every 5 fs. The data is saved for every $25 \mathrm{ps}$ for subsequent analyses. 


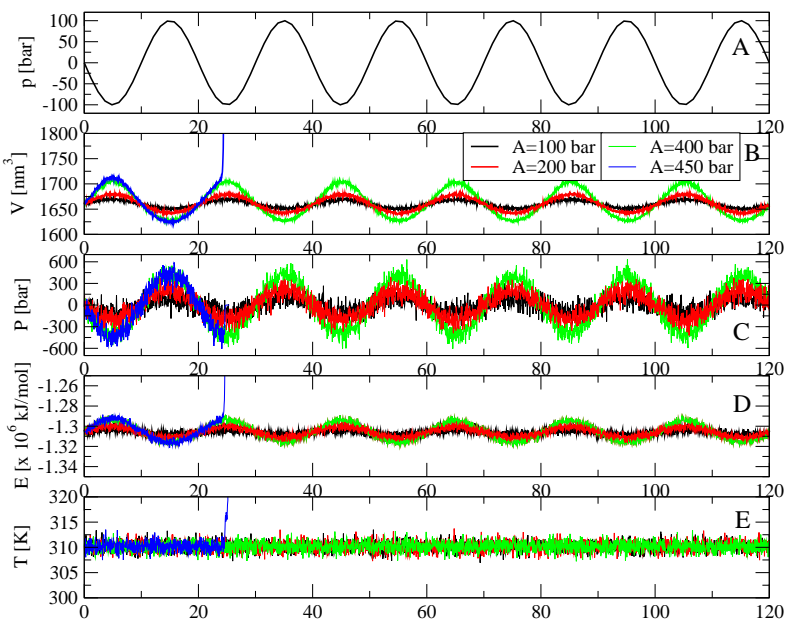

Figure 2: Time evolution of various quantities, including the ultrasound (A), the system volume (B), the pressure (C), the potential energy (D) and the temperature (E). Shown are results obtained with different ultrasound intensities $A=$ $100,200,400$ and 450 bar. The period of the ultrasound is $\tau=20 \mathrm{~ns}(50 \mathrm{MHz})$.

\section{RESULTS}

\section{A. Response of the system to the ultrasound}

In all simulations, the ultrasound period $\tau=20 \mathrm{~ns}$ $(50 \mathrm{MHz})$ is used, and the amplitude is varied in the range $A=100-500$ bar. To obtain a first impression, Fig.2(A) shows, as an example, the time evolution of the ultrasound $p(t)$ with amplitude $A=100$ bar. The negative and positive periods are called the rarefaction and compression phases, respectively. For each ultrasound amplitude, the simulation is run for 120 ns, i.e. the system is excited by five ultrasound pulses, and the response of the system to the ultrasound is shown in Fig. 2. Let us first present the results for the case $A=400$ bar. During the first rarefaction phase, i.e. $p(t) \leq 0(t \leq \tau / 2)$, the system is expanded with an increase in the volume from the initial value $V \sim 1650 \mathrm{~nm}^{3}$ to $V \sim 1700 \mathrm{~nm}^{3}$ at $5 \mathrm{~ns}$ [Fig.2(B)]. As a consequence, the pressure inside the system becomes negative, and decreases from the initial equilibrium value of 1 bar to -400 bar [Fig.2(C)]. As a result, atoms are pulled apart, thus the attractive interactions, including the long-ranged Coulombic and van der Waals interactions become dominant. Thus the potential energy of the system is decreased from $-1.30 \times 10^{6}$ to $-1.29 \times 10^{6} \mathrm{~kJ} / \mathrm{mol}$ [Fig.2(D)]. Because the system is coupled to the heat bath, its temperature is always fluctuating around the reference value of $300 \mathrm{~K}$ [Fig.2(E)]. Within the time interval $\tau / 4 \leq t \leq \tau / 2$, the ultrasound intensity is reduced, but still in the rarefaction phase, thus the system is relaxed, as indicated by the decrease in the volume, pressure and potential energy, and achieved the equilibrium state at $t=\tau / 2=10 \mathrm{~ns}$. Next, the ultrasound is in the compression phase, i.e. $p(t) \geq 0$ $(\tau / 2 \leq t \leq \tau)$, the system is compressed as shown by the decrease and increase in the volume and pressure, respectively. The atoms are pushed closely, thus the short-ranged Coulombic and van der Waals interactions are dominated, therefore the potential energy of the system is increased from $-1.30 \times 10^{6}$ to $-1.31 \times 10^{6} \mathrm{~kJ} / \mathrm{mol}$ [Fig.2(D)]. After $t \geq 3 \tau / 4$, the ultrasound intensity is reduced, but still in the compression phase, the system volume is increased, the pressure is reduced and the potential energy is increased. At $t=\tau$, the ultrasound is vanished and the system reaches the equilibrium state, finishing one ultrasound irradiation period. The response of the system is repeated for the next four ultrasound periods. As seen from Fig.2, the response of the system to the weaker ultrasounds with $A=100$ or 200 bar, is similar to that of $A=400$ bar, but less intense. However, by increasing the ultrasound intensity to $A=450 \mathrm{bar}$, the system is able to oscillate with the ultrasound within the first period ( $t \leq 20 \mathrm{~ns}$ ), but then exploded during the second ultrasound period. That is, at $t=25 \mathrm{~ns}$, when the ultrasound is fully expanded, the system volume suddenly increases to a large value, the pressure is reduced immediately to zero, and the potential energy becomes very large because atoms are largely separated apart. Although the system is coupled to the heat bath, as the expansion takes place very fast, the velocity of atoms are not rapidly rescaled and the temperature of the system is significantly increased. Technically, the simulation is crashed.

\section{B. Response of the claudin-5 tight junction to the ultrasound}

To understand the effect of ultrasound on the claudin5 tight junction, we calculate the root-mean-square displacement (RMSD) of two cis-dimers (each anchored to each membrane) with respect to the initial structure for different ultrasound intensities. As seen from Fig.3(A), the RMSDs undergo small increases of $\sim 0.25 \mathrm{~nm}$ compared to the initial structure, despite large variations in the ultrasound intensities from 100 to 450 bar. Interestingly, although the system is exploded with $A=450$ bar as shown above, but the RMSD does not change much, indicating that the overall structure of the tight junction is well-maintained. Fig.3(B) shows the total number of the intermolecular hydrogen bonds (Hbond) between two dimers. We observe that the number of Hbond fluctuates in time, but it does not follow the oscillation of the ultrasound pressure. After $120 \mathrm{~ns}$, two dimers are slightly separated, as indicated by a small reduction in the number of Hbond from 5 to 3 for $A=400$ bar. Even at 450 bar where the system is exploded, there is no large change in the number of Hbond, indicating that the tight junction is not disrupted.

Next, we wish to understand the impact of the ultra- 

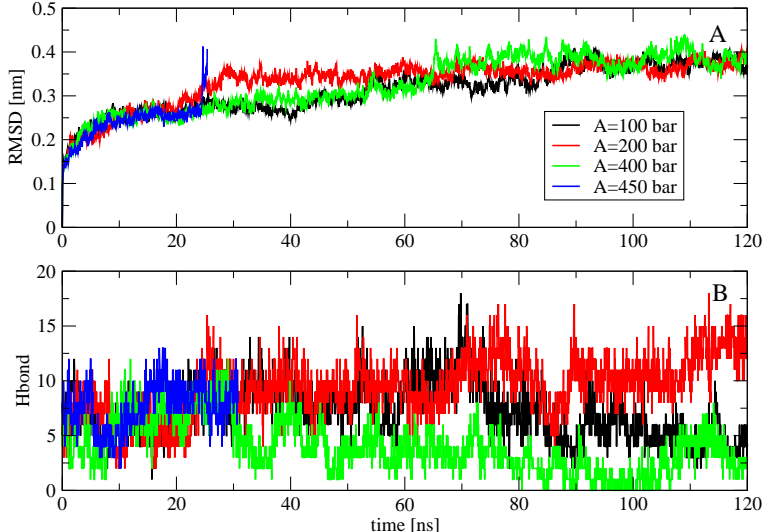

Figure 3: The time evolution of the RMSD of the claudin-5 tight junction with respect to the initial structure (A), and the total number of intermolecular Hbonds between two dimers (B). Shown are results obtained from simulations using ultrasound with period of $20 \mathrm{~ns}$ and various intensities.
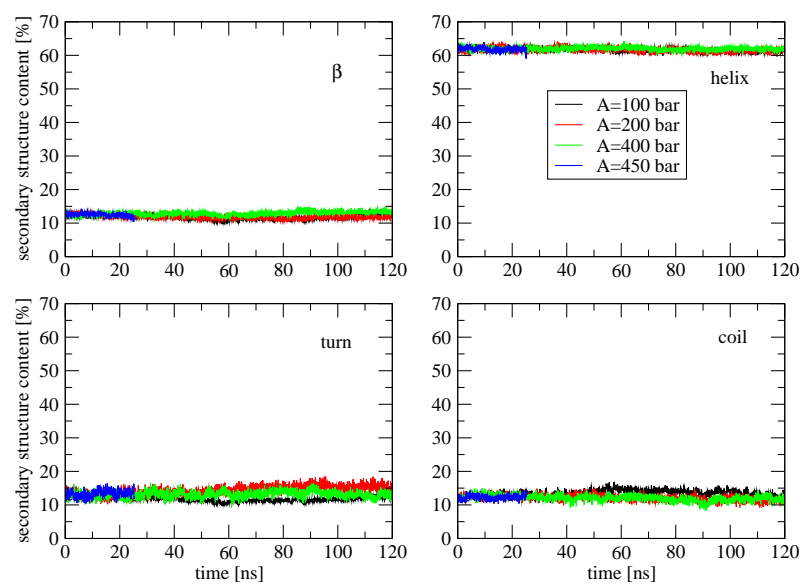

Figure 4: The time evolution of the population of various secondary structures. For each structure, shown is the total populations of four claudin-5 proteins pertaining to the junction. Shown are results obtained from simulations using ultrasound with period of $20 \mathrm{~ns}$ and various intensities.

sound on the secondary structures of the claudin-5 proteins. To this end, we calculate the population of the secondary structures of two dimers by using the STRIDE program $[65]$ and the results are shown in Fig.4. As seen, the extracellular loops of claudin- 5 proteins, which are mainly in the $\beta$ structure, contribute initially to $\approx 15 \%$ of the secondary structure population. The transmembrane helix bundles contribute to $\approx 65 \%$, and $\approx 20 \%$ are attributed to the turn and coil structures of the linkers and of the intracellular loops. We observe that the sec-
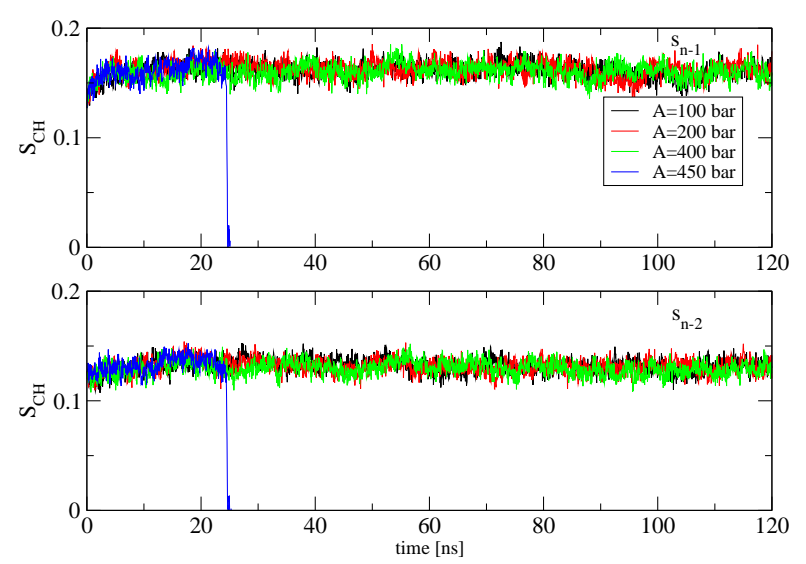

Figure 5: Time evolution of the carbon-hydrogen order parameters of the first $s_{n-1}$ (upper) and second $s_{n-2}$ lipid tails, respectively (lowver). These results are averaged over 15 and 17 order parameters of $\mathrm{C}-\mathrm{H}$ vectors of the first and second lipid tails, respectively, and obtained from simulations using ultrasound with period of $20 \mathrm{~ns}$ and various intensities.

ondary structures hardly undergo any changes, irrespective large variations in the ultrasound intensities, even at 450 bar where the system is exploded.

All these results show that both $3 \mathrm{D}$ and 2D structures of individual claudin- 5 proteins, and of the whole tight junction are largely maintained, and the tight junction is not disrupted by the ultrasound irradiation.

\section{Response of the lipid bilayer membranes to ultrasound}

To investigate the response of the lipid bilayers to the ultrasound, we first calculate the carbon-hydrogen order parameters of the lipid tails $S_{\mathrm{CH}}=\left\langle 3 \cos ^{2} \theta-1\right\rangle / 2$, where $\theta$ is the angle between a $\mathrm{C}-\mathrm{H}$ bond vector and the bilayer normal. The angular brackets represent molecular and temporal ensemble averages. For a POPC lipid, the number of $\mathrm{C}-\mathrm{H}$ vectors in the first, $\mathrm{s}_{n-1}$, and second, $\mathrm{s}_{n-2}$, tails are 15 and 17 , respectively. To obtain an overall picture on the order of each tail, we simply take the average of the order parameters of all $\mathrm{C}-\mathrm{H}$ vectors pertaining to that tail. The results are shown in Figs. 5 for two tails. As seen, for the ultrasound intensities $A=100,200$ and 400 bar, the order parameters of both tails do not undergo any oscillation with ultrasounds, indicating that lipids are quite ordered. However, with $A=450$ bar, the order parameters drop to zeros at $t=25 \mathrm{~ns}$, a moment when the system is exploded, indicating significantly structural changes in the membranes. To understand this, we calculate the shortest intra-distance between two monolayers of each bilayer, and the shortest inter-distance between two bilayer membranes. The latter measures the gap 


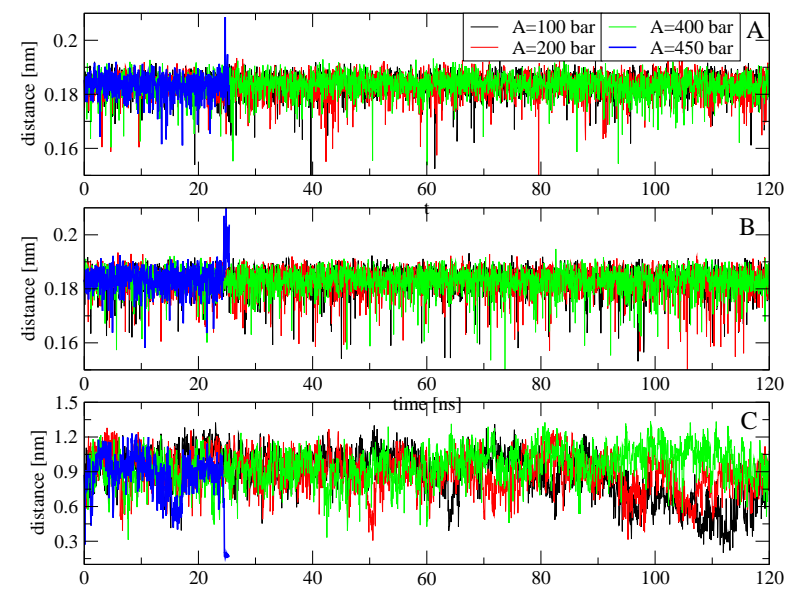

Figure 6: Time evolution of the shortest intra-distances between two monolayers of the first (A) and second (B) cell membrane lipid bilayers. The shortest inter-distance between two lipid bilayer cell membranes is shown in the (C). Shown are results obtained from simulations using ultrasound with period of $20 \mathrm{~ns}$ and various intensities.

of the tight junction between two cells. The results are shown in Fig.6. As seen, two monolayers of individual lipid bilayer membranes are always separated by intradistances of $\approx 0.18 \mathrm{~nm}$ [Figs.6(A), (B)], and two lipid bilayer membranes of two adjunct cells are stay intact at $\approx 0.9 \mathrm{~nm}$ for ultrasounds with $A=100,200$ and 400 bar. A visual inspection of a snapshot at 120 ns of the trajectory with $A=400$ bar shown in Fig.7(A) confirms these results, that is the membranes and the claudin- 5 tight junction structures are well-maintained, and similar to the initial structures shown in Fig.1(B). However, with $A=450$ bar, we observe that the intra-distances between two monolayers of individual bilayers increase and become suddenly large at $t=25$ ns when the system is exploded. At the same time, the inter-distance between two bilayer membranes is reduced from 0.9 to $0.5 \mathrm{~nm}$. To understand this, we show in Fig. 7 two snapshots just before collapsing at $t=24.5$ and $24.7 \mathrm{~ns}$, and one snapshot at the collapsed moment at $25 \mathrm{~ns}$. We observe that when the system is about to explode, the two monolayers of individual membranes are separated apart, and this pushes two lipid bilayer membranes of two cells to each others [Figs.7(B),(C)] . When the system is fully exploded, two monolayers are highly separated, two cells get closer, and lipids become disorder. This explains the reduction in the order parameters shown in Fig.5. Nevertheless, the claudin- 5 tight junction is hardly affected, and still maintained by two monolayers of two adjunct cells [Fig.7(D)]

\section{DISCUSSION AND CONCLUDING REMARK}

In the HIFU experiments the direct interaction of ultrasound with the $\mathrm{BBB}$ is the cause of the $\mathrm{BBB}$ disruption. For the LIFU experiments, it is widely believed that ultrasound induces the stable and/or inertial cavitation of bubble, which in turn exerts acoustic shear forces and/or shockwave on the BBB, leading to the BBB opening. However, in principle, ultrasound also interacts directly with the cells and tight junction. This results in complicated $\mathrm{BBB}$ opening mechanisms, especially at the molecular level. Thus, our strategy is to split this complex problem into simpler tasks. In this work, our primary aim is to understand how the lipid bilayer cell membrane and the tight junction respond to the ultrasound and whether the cell membrane and/or the claudin-5 junction are affected by ultrasound. Before discussing the results, the limitations of our study should be pointed out. First, our BBB model is very simple, composed of only two lipid bilayers, mimicking two portions of neighbouring cells. The tight junction is composed of a paracellular pore formed by two cis-dimers of claudin-5 proteins. Nevertheless, given the fact that currently there is no experimental molecular structure of the BBB, this computational model still can serve as a starting point for MD simulations, which can provide some insights into the BBB opening mechanism. Second, the experimental frequency of the ultrasound is usually tuned between $\omega=0.5-50 \mathrm{MHz}$, the ultrasound pressure is in the range $10-50$ bar for LIFU experiments[66, 67], and $100-1000$ bar for HIFU experiments[68]. The ultrasound irradiation duration is usually in the second to minute timescales[66,67]. To be close to experiments, we use an ultrasound frequency of $50 \mathrm{MHz}$ for our simulations. To be computationally feasible with current computer technology, the simulation time can only be in the nanosecond scale. This means that our system is only irradiated by several ultrasound cycles. We use the typical HIFU intensity in the range 100-500 bar for our simulations, which are strong enough to allow us to obtain response of the BBB within the simulation timescales. For low intensities used in LIFU experiments, the timescale of simulations would be much longer to obtain any significant responses of the BBB, and this is beyond our current capability.

Our simulations show that with weak ultrasound intensities $A \leq 400$ bar, the structures of both lipid cell membranes and claudin- 5 tight junction are well-maintained. This implies that for the HIFU experiments, the direct interaction of ultrasound with BBB does not yield to the opening neither at the cells nor tight junction. In the context of the LIFU experiments, where the ultrasound intensity is even weaker, this interaction can be safely excluded in the interpretation of the BBB opening mechanism, confirming that the interaction of the bubble cavitation with BBB is the main cause as hypothesised. With stronger ultrasound intensities, say $A=450$ bar, 

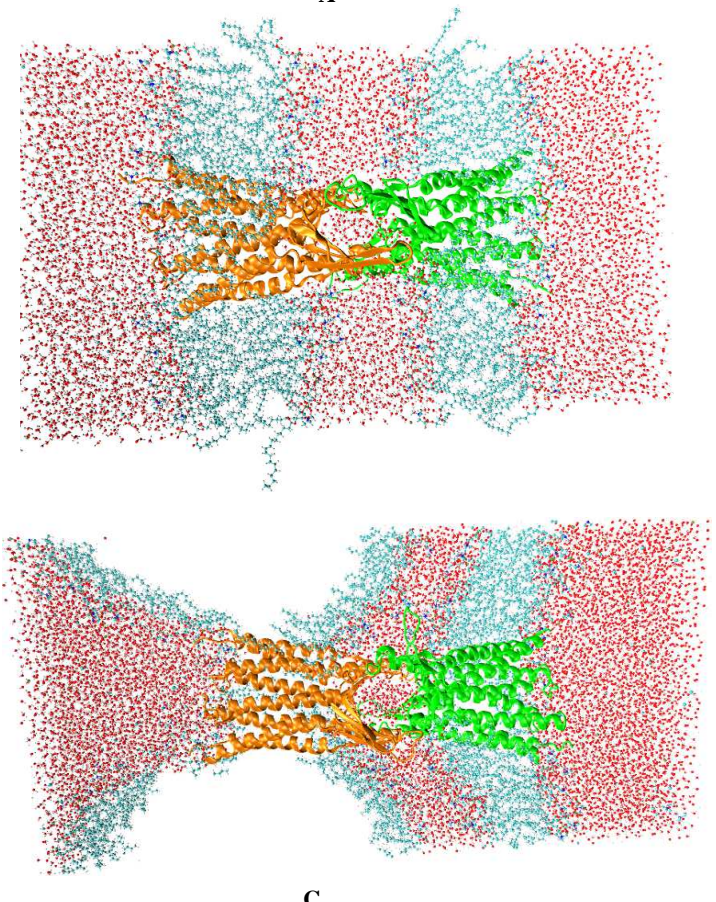
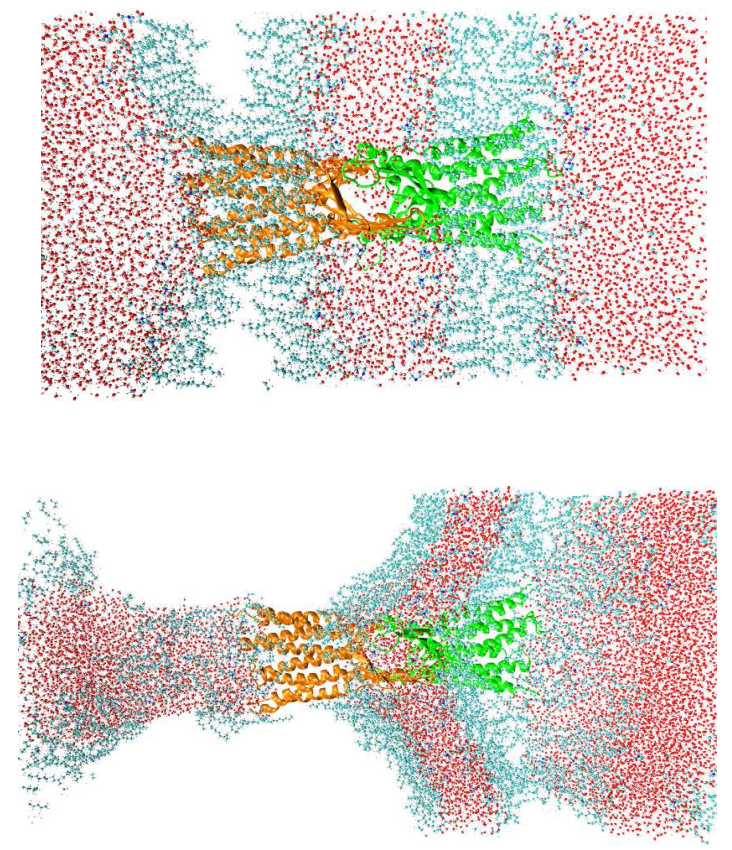

D

Figure 7: Snapshots of the system at 120 ns obtained by using ultrasound intensity of 400 bar (A), and at $24.5,24.7$ and 25 ns obtained by using ultrasound intensity of 450 bar (B, C, D respectively). The ultrasound period of 20 ns is used in all simulations. While the bilayers are disrupted, the tight junction is well-maintained.

we observe that two monolayers within individual lipid bilayers are detached following the expansion of the simulation box during the ultrasound rarefaction phase, and this could result in the damage of the cell membrane. In contrast, the tight junction between two cells is hardly affected. This suggests that the BBB disruption likely takes place at the cell surface instead of at the cell junction.

To explain this, we calculate the interaction potential energy acting on two monolayers M1 and M2 of a bilayer shown in Fig.8. As seen, the monolayer M1 is stabilised by potential energy $\mathrm{V}(\mathrm{M} 1)$ which is contributed by four interactions $\mathrm{V}(\mathrm{M} 1)=\mathrm{V}(\mathrm{M} 1-\mathrm{C} 1)+\mathrm{V}(\mathrm{M} 1-\mathrm{W} 1)+\mathrm{V}(\mathrm{M} 1-$ $\mathrm{C} 2)+\mathrm{V}(\mathrm{M} 1-\mathrm{M} 2)$, and the monolayer M2 is stabilised by three interactions $\mathrm{V}(\mathrm{M} 2)=\mathrm{V}(\mathrm{M} 2-\mathrm{C} 1)+\mathrm{V}(\mathrm{M} 2-\mathrm{W} 1)+$ $\mathrm{V}(\mathrm{M} 2-\mathrm{M} 1)$, where $\mathrm{V}(\mathrm{M} 1-\mathrm{C} 1)$ is the interaction potential energy between the monolayer M1 and the claudin cisdimers $\mathrm{C} 1$ embed in $\mathrm{M} 1, \mathrm{~V}(\mathrm{M} 1-\mathrm{W} 1)$ is the interaction energy between M1 and waters between two cells, and so on. Fig.8 shows the time evolution of V(M1) and V(M2) for an example case $A=400$ bar. As seen, these potential energies oscillate with the ultrasound, i.e, their values increase and decrease using the ultrasound rarefaction and compression phases, respectively. However, on average, $\mathrm{V}(\mathrm{M} 1)$ is $\sim-2000 \mathrm{~kJ} / \mathrm{mol}$ lower than $\mathrm{V}(\mathrm{M} 2)$, suggesting that the monolayer M1 is more stable than M2. Therefore, while the M1 layer is anchored by the tight junction, the M2 layer moves following the expansion of the system during the ultrasound rarefaction phase, resulting in the detachment of the layer M2 from the layer M1.

In the real $\mathrm{BBB}$, the tight junction is much more complicated, composed of many claudin-5 proteins and other proteins. Of course, this will increase the tightness of the junction but also increase the interaction between cell membranes with transmembrane proteins of the tight junction. The question is then whether the BBB is still disrupted at the cell membrane position? To this end, we construct an empirical BBB model whose tight junction is composed of four pairs of two cis-dimers, each pair is located on the corner of a square with edge lengths of 10 nm [Fig.9]. This is obtained by translating a pair of two cis-dimers of the above model [Fig.1] in the membrane surface for $10 \mathrm{~nm}$ along the $\mathrm{x}$ and $\mathrm{y}$-axis. We then carry out simulations using the same parameters as used for the previous model. Fig.9 shows snapshots after six ultrasound irradiation periods, i.e. at $120 \mathrm{~ns}$ for $A=400$ and 450 bar. Consistent with the results of the single cis-dimer pair model, the structure of the four cis-dimer pairs model is well-maintained with $A=400$ bar, but with $A=450$ bar, two monolayers of individual bilayer membranes is detached, suggesting that the BBB is also damaged at the cell plasma membrane rather than at the tight junction.

Let us discuss how the separation of monolayers of the cell membrane results in the enhancement of the drug permeability. The separation of two monolayers in a bilayer results in large spaces at the middle of the lipid bilayer (Figs.7(B),(C), (D)). This phenomenon has been 

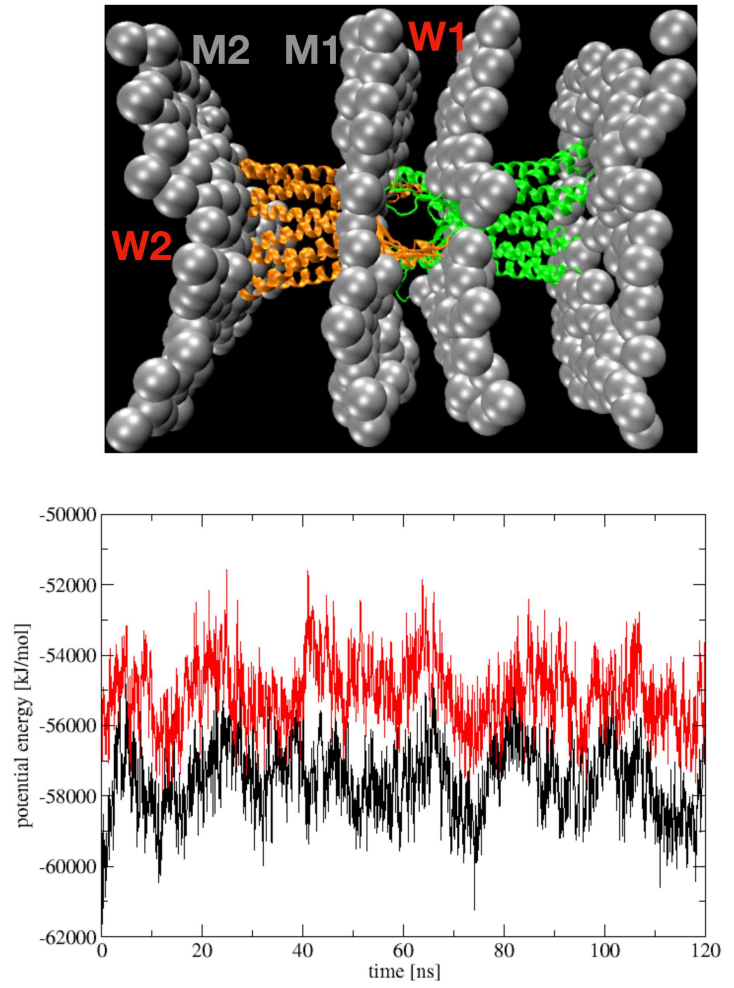

Figure 8: (Upper) A structure of the BBB model where components are labeled as M1 and M2 for two monolayers of a cell membrane bilayer; W1 and W2 for waters between two bilayers and above the membrane $\mathrm{M} 2$, respectively; $\mathrm{C} 1$ and C2 for two claudin-5 dimers embed in two cell membrane lipid bilayers. (Lower) Time evolution of the potential energy acting on M1 (black) and M2 (red). For clarity, only phosphate groups of the lipids are shown in beads and waters are not shown.

observed experimentally, in the context of drug delivery studies using liposomes. That is, under LIFU irradiation, gas bubble nuclei may be formed in the hydrophobic region of the lipid bilayer of the liposomes. These nuclei grow until they permeate the membrane, forming a transient pore through which the drug is released $[69,70]$. At the molecular level, the presence of the large spaces alternates the diffusive process of drugs across the membrane. Indeed, a number of simulations have shown that the free energy profile for translocation across the lipid bilayer of drugs usually exhibits a high barrier at the middle of the two monolayers[71-74]. If two monolayers are separated, then this leaves a large space in the middle of the bilayer, resulting in high population of drugs in this area, i.e. the free energy barrier is reduced, and therefore the drug permeability is increased. We find that the molecular mechanism of the ultrasound induced permeability of $\mathrm{BBB}$ is similar to that observed in liposomes, but the origin of the large spaces formation may be different. It is expected that considerable heterogeneity exists in the preparations of liposomes, and it is unlikely that all li- posomes are unilamellar. Thus, large spaces are likely formed at the flexible areas of the membrane of liposomes. For the BBB, because the outer layer of the cell membrane is anchored by the tight junction, but the inner layer is contracted during the rarefaction phase of the ultrasound, therefore this separation results in large spaces inside the cell membrane bilayer [Fig.8].

As reviewed earlier in this paper, under HIFU irradiation, several experiments observed the BBB disruption within the damaged areas[16, 17], but other results showed the BBB disruption without damage of the brain parenchyma $[18,19]$. In both cases, it was unclear whether the $\mathrm{BBB}$ disruption is due the cell damage or opening of the tight junction[11]. Our simulations could suggest that the BBB may be disrupted due to the damaged of the cells, but the tight junction is unlikely happened.

A large body of literature confirms that LIFU-induced BBB opening involves at least four mechanisms: transcythosis, transendothelial opening, i.e. fenestration and pore formation, tight junction opening at low ultrasound intensities, and passage through damaged cell at high ultrasound intensities[20, 75]. Our simulation study suggests that with low ultrasound intensities used in LIFU experiments, the impact of the direct interactions of ultrasound with cells and with tight junctions can be negligible. Thus, the interaction between bubble and the BBB plays an essential role. Indeed, it has been suggested that the acoustic microstreaming generated by stable bubble cavitation stimulates the transcythosis, transendothelial opening, and the inertial cavitation may induce cell membrane pore formation. The tight junction opening may due to the volume expansion of microbubbles in the vessel, which in turn exerts direct mechanical forces on the BBB. However, at high ultrasound intensity, the direct interaction of ultrasound with the BBB becomes significant, leading to the cell damage as observed by experiments[20, 75], and confirmed by our simulations.

In conclusions, we have performed a molecular dynamics simulation study to understand the molecular impacts of HIFU and LIFU on the BBB opening. The study shows that even with a relatively high ultrasound intensity of 400 bar, the structures of the cell membranes and tight junction were well-maintained, implying that the direct interaction between LIFU with the BBB is not responsible for the BBB opening, that has been observed experimentally. At higher intensities ( $A \geq 450$ bar), the rarefaction of ultrasound pulls two monolayers of individual cell membrane lipid bilayers apart, creating air compartments inside the bilayer. This reduces the free energy barrier for translocation across the lipid bilayer of drugs, thus enhances the drug permeability. At very high intensities, two monolayers are largely separated, resulting in cell damage, implying that the $\mathrm{BBB}$ is primarily disrupted at the damaged areas as observed by experiment. The MD simulations of the full system composed of ultrasound, bubbles and $\mathrm{BBB}$ will be carried out to understand impacts of bubble cavitation on the 

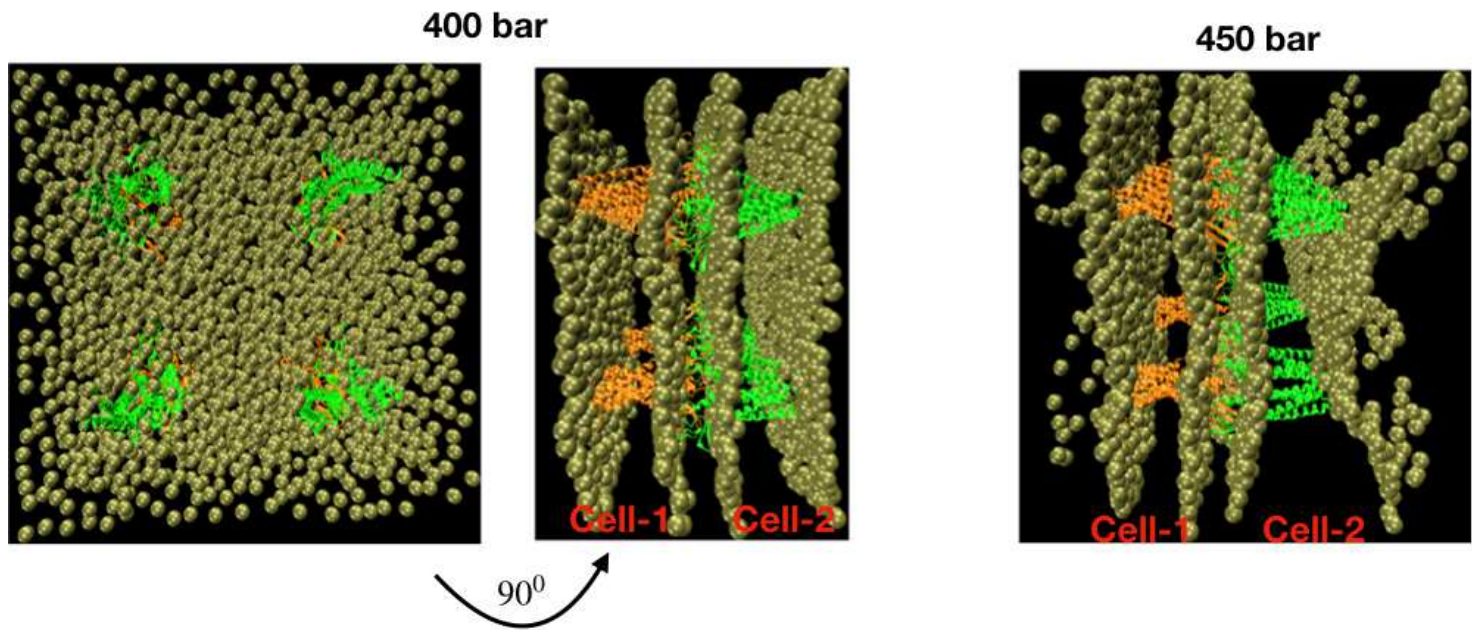

Figure 9: Snapshots of the system whose tight junction is composed of four pairs of claudin- 5 cis-dimers at 120 ns obtained by using ultrasound intensity of 400 bar (left), and at $25 \mathrm{~ns}$ obtained by using ultrasound intensity of 450 bar (right). The ultrasound period is $20 \mathrm{~ns}$ in all simulations. Waters are not shown for clarity. The separation of two monolayers is clearly seen from the snapshot at 450 bar. For clarity, only phosphate groups of the lipids are shown in beads and waters are not shown.

BBB model. Also, more detailed BBB models will be constructed. These works may open the door of drugs delivery for Alzheimer's diseases[76, 77].

Acknowledgments This work has been supported by the Department of Science and Technology at Ho Chi Minh City, Vietnam (grant 10/2018/HDKHCNTT), CNRS, the World Bank and the Vietnam Ministry of Science and Technology FIRST project (grant 13/FIRST/1.a/VNU1), the Polish Narodowym
Centrum Nauki (NCN, grant 2019/35/B/ST4/02086), the National Science Foundation (NSF, grant SI2SEE-1534941), the National Institute of Health (NIHR01GM118508) and the CINES center for providing computer facilities (project A0080711440).

DATA AVAILABILITY The data that support the findings of this study are available from the corresponding author upon reasonable request.
[1] Daneman, R.; Prat, A. The blood-brain barrier. Cold Spring Harb. Perspect. Biol. 2015, 7, a020412.

[2] Janigro, D. Are you in or out? Leukocyte, ion, and neurotransmitter permeability across the epileptic blood-brain barrier. Epilepsia 2012, 53, 26-34.

3] Vykhodtseva, N.; McDannold, N.; Hynynen, K. Progress and problems in the application of focused ultrasound for blood-brain barrier disruption. Ultrasonics 2008, 48, 279-296.

[4] Pardridge, W. M. Molecular Trojan horses for bloodbrain barrier drug delivery. Discov Med. 2006, 6, 139143.

[5] Gross, R. E.; Watts, R. L.; Hauser, R. A.; et. al., Intrastriatal transplantation of microcarrier-bound human retinal pigment epithelial cells versus sham surgery in patients with advanced Parkinson's disease: a double-blind, randomised, controlled trial. Lacet Neurol. 2011, 10, 509.

[6] Marks, J. W. J.; Ostrem, J. L.; Verhagen, L.; et. al, Safety and tolerability of intraputaminal delivery of CERE-120 (adeno-associated virus serotype 2-neurturin) to patients with idiopathic Parkinson's disease: an openlabel, phase I trial. Lacet Neurol. 2008, 7, 400.

[7] Pardridge, W. M.; Boado, R. J. Reengineering biopharmaceuticals for targeted delivery across the blood-brain barrier. Methods Enzymol 2012, 503, 269.
[8] Matsukado, K.; Sugita, M.; Black, K. L. Intracarotid low dose bradykinin infusion selectively increases tumor permeability through activation of bradykinin B2 receptors in malignant gliomas. Brain Res. 1998, 792, 10-15.

[9] Rapoport, S. I. Functional brain imaging to identify affected subjects genetically at risk for Alzheimer's disease. Proc Natl Acad Sci USA 2000, 97, 5696-5698.

[10] Hynynen, K.; McDannold, N.; Vykhodtseva, N.; Jolesz, F. A. Noninvasive MR imaging-guided focal opening of the blood-brain barrier in rabbits. Radiology 2001, 220, 640 .

[11] Mesiwala, A. H.; Farrell, L.; Wenzel, H. J.; Silbergeld, D. L.; Crum, L. A.; Winn, H. R.; Mourad, P. D. High-intensity focused ultrasound selectively disrupts the blood-brain barrier in vivo. Ultrasound Med. Biol. 2002, 28, 389-400.

[12] B.Baseri,; Choi, J. J.; Tung, Y.; Konofagou, E. E. Multi-Modality Safety Assessment of Blood-Brain Barrier Opening Using Focused Ultrasound and Definity Microbubbles: A Short-Term Study. Ultrasound Med Biol. 2010, 36, 1445-1459.

[13] McDannol, N.; Arvanitis, C. D.; Vykhodtseva, N.; Livingstone, M. S. Temporary disruption of the blood-brain barrier by use of ultrasound and microbubbles: safety and efficacy evaluation in rhesus macaques. Cancer Res. 
2012, 72, 3652-63

[14] Horodyckid, C.; Canney, M.; Vignot, A.; Boisgard, R.; Drier, A.; Huberfeld, G.; Francois, C.; Prigent, A.; Santin, M.; C.Adam,; Willer, J. C.; Lafon, C.; Chapelon, J. Y.; Carpentier, A. Safe long-term repeated disruption of the blood-brain barrier using an implantable ultrasound device: a multiparametric study in a primate model. J Neurosurg. 2016, 10, 1-11.

[15] Barnard, J. W.; Fry, W. J.; Fry, F. J.; Krumins, R. F. Effects of high intensity ultrasound on the central nervous system of the cat. J Comp Neurol. 1995, 103, 459-484.

[16] Bakay, L.; Ballantine, J. H. T.; Hueter, T. F.; et. al, Ultrasonically produced changes in the blood brain barrier. Arch. NeurPsych. 1956, 76, 457-467.

[17] Patrick, J. T.; Nolting, M. N.; Goss, S.; Dines, K.; Clendenon, J.; Rea, M.; Heimburger, R. Ultrasound and the blood-brain barrier. Adv Exp Med Biol. 1990, 267, 369381.

[18] Ballantine, J. H.; Bell, E.; Manlapaz, J. Progress and problems in the neurological applications of focused ultrasound. J Neurosurg. 1960, 17, 858-876.

[19] Vykhodtseva, N.; Hynynen, K.; Damianou, C. Histologic effects of high intensity pulsed ultrasound exposure with subharmonic emission in rabit brain in vivo. Ultrasound Med. Biol. 1995, 21, 967-979.

[20] Sheikov, N.; McDannold, N.; Vykhodtseva, N.; Jolesz, F.; Hynynen, K. Cellular mechanisms of the blood-brain barrier opening induced by ultrasound in presence of microbubbles. Ultrasound Med. Biol 2004, 30, 979-989.

[21] Choi, J. J.; Small, S. A.; Konofagou, E. E. Optimization of Blood-Brain Barrier Opening in Mice using Focused Ultrasound. IEEE Ultrasonics Symposium 2006, 540-543.

[22] Hynynen, K.; McDannold, N.; Sheikov, N. A.; Jolesz, F. A.; Vykhodtseva, N. Local and reversible blood-brain barrier disruption by noninvasive focused ultrasound at frequencies suitable for trans-skull sonications. Neuroimage 2005, 24, 12-20

[23] O'Reilly, M. A.; Huang, Y.; Hynynen, K. Impact of standing wave effects on transcranial focused ultrasound disruption of the blood-brain barrier in a rat model. Phys. Med. Biol. 2010, 55, 5251-5267.

[24] Sheikov, N.; McDannold, N.; Sharma, S.; Hynynen, K. Effect of focused ultrasound applied with an ultrasound contrast agent on the tight junctional integrity of the brain microvascular endothelium. Ultrasound Med Biol. 2008, 34, 1093.

[25] Vlachos, F.; Tung, Y.; Konofagou, E. E. Permeability assessment of the focused ultrasound-induced blood-brain barrier opening using dynamic contrast-enhanced MRI. Phys. Med. Biol. 2010, 55, 5451-5466.

[26] Vlachos, F.; Tung, Y.; Konofagou, E. E. Permeability dependence study of the focused ultrasound-induced bloodbrain barrier opening at distinct pressures and microbubble diameters using DCE-MRI. Mag. Reson. Med. 2011, $66,821-830$.

[27] Chen, P. et al. Novel magnetic/ultrasound focusing system enhances nanoparticle drug delivery for glioma treatment. Neuro-Oncology 2010, 12, 1050-1060.

[28] Liu, H. L.; Hua, M.; Chen, P.; Chu, P.; Pan, C.; Yang, H.; Huang, C.; Wang, J.; Yen, T.; Wei, K. Blood-brain barrier disruption with focused ultrasound enhances delivery of chemotherapeutic drugs for glioblastoma treatment. Radiology 2010, 255, 415-425.
[29] Jordao, J.; Ayala-Grosso, C. A.; Markham, K.; Huang, Y.; Chopra, R.; McLaurin, J.; Hynynen, K. Aubert, I. Antibodies Targeted to the Brain with ImageGuided Focused Ultrasound Reduces Amyloid- $\beta$ Plaque Load in the TgCRND8 Mouse Model of Alzheimer's Disease. PloS One 2010, 5, e10549.

[30] Raymond, S. B.; Treat, L. H.; Dewey, J. D.; McDannold, N. J.; Hynynen, K.; Bacskai, B. J. Ultrasound Enhanced Delivery of Molecular Imaging and Therapeutic Agents in Alzheimer's Disease Mouse Models. PloS One 2008, 3, e2175.

[31] Nhan, T.; Burgess, A.; Lothar, L.; Hynynen, K. Modeling localized delivery of Doxorubicin to the brain following focused ultrasound enhanced blood-brain barrier permeability. Phys. Med. Biol. 2014, 59, 5987- 6004.

[32] Wiedemair, W.; Tukovic, Z.; Jasak, H.; Poulokakos, D.; Kurtcuoglu, V. On Ultrasound-Induced Microbubble Ossillation in a Capillary Blood Vessel and its Implications for the Blood-Brain Barrier. Phys. Med. Biol. 2012, 57 , 1019-1045.

[33] Hosseinkhah, N.; Goertz, D. E.; Hynynen, K. Microbubbles and Blood-Brain Barrier Opening: A Numerical Study on Acoustic Emissions and Wall Stress Predictions. IEEE Trans Biomed Eng. 2015, 62, 1293-1304.

[34] Hosseinkhah, N.; Hynynen, K. A Three-Dimensional Model of an Ultrasound Contrast Agent Gas Bubble and its Mechanical Effects on Microvessels. Phys Med Biol. 2012, 57, 785-800.

[35] Hosseinkhah, N.; Chen, H.; Matula, T.; Burns, P. N.; Hynynen, K. Mechanisms of Microbubble-Vessel Interactions and Induced Stresses: A Numerical Study. J Acoust Soc Am. 2013, 134, 1875-1885.

[36] Qin, S.; Ferrara, K. W. Acoustic response of compliable microvessels containing ultrasound contrast agents. Phys Med Biol. 2006, 51, 5065-5088

[37] Wiedemair, W.; Tukovic, Z.; Jasak, H.; Poulikakos, D. Kurtcuiglu, V. The breakup of intravascular microbubbles and its impact on the endothelium. Biomech. Model Mechaobiol 2016, DOI:10.1007/s102337-016-0840-z.

[38] Ganzenmuller, G. C.; Hiermaier, S.; Steinhauser, M. . O. ShockWave Induced Damage in Lipid Bilayers: A Dissipative Particle Dynamics Simulation Study. Soft Matter 2011, 7, 4307-4317.

[39] Choubey, A.; Vedadi, M.; Nomura, K.; Kalia, R. K.; Nakano, A.; Vashishta, P. Poration of Lipid Bilayers by Shock-Induced Nanobubble Collapse. Appl. Phys. Lett 2011, 98, 023701.

[40] Schanz, D.; Metten, B.; Kurz, T.; Lauterborn, W. Molecular Dynamics Simulations of Cavitation Bubble Collapse and Sonoluminescence. New J. Phys. 2012, 14 113019 .

[41] Nomura, A. S. K.; Kalia, R. K.; Nakano, A.; Vashishta, P. Nanobubble Collapse on a Silica Surface in Water: Billion-Atom Reactive Molecular Dynamics Simulations. Phys. Rev. Lett. 2013, 111, 184503.

[42] Koshiyama, K.; Kodama, T.; Yano, T.; Fujikawa, S. Structural Change in Lipid Bilayers and Water Penetration Induced by Shock Waves: Molecular Dynamics Simulations. Biophys J. 2006, 91, 2198-2205.

[43] Santo, K. P.; Berkowitz, M. L. Shock Wave Induced Collapse of Arrays of Nanobubbles Located Next to a Lipid Membrane: Coarse Grained Computer Simulations. J. Phys. Chem. B 2014, 119, 8879-8889.

[44] Santo, K. P.; Berkowitz, M. L. Shock Wave Interaction 
with a Phospholipid Membrane: Coarse-Grained Computer Simulations. J. Chem. Phys. 2014, 140, 054906.

[45] Fu, H.; Comer, J.; Cai, W.; Chipot, C. Sonoporation at Small and Large Length Scales: Effect of Cavitation Bubble Collapse on Membranes. J Phys Chem Lett. 2015, 5, 413-418.

[46] Adhikari, U.; Goliaei, A.; Berkowitz, M. L. Mechanism of membrane poration by shock wave induced nanobubble collapse: a molecular study. J. Phys. Chem. B 2015, 119, 6225 .

[47] Agrawal, V.; Peralta, P.; Li, Y.; J. Oswald, ., J. Chem. Phys. 145 A pressure-transferable coarse-grained potential for modeling the shock Hugoniot of polyethylene. $J$. Chem. Phys. 2016, 145, 104903.

[48] Goliaei, A.; Adhikari, U.; Berkowitz, M. L. Opening of the Blood-Brain Barrier Tight Junction Due to Shock Wave Induced Bubble Collapse: A Molecular Dynamics Simulation StudyJ. ACS Chem. Neurosci 2015, 6, 1296.

[49] Viet, M. H.; Derreumaux, P.; Nguyen, P. H. Nonequilibrium All-Atom Molecular Dynamics Simulation of the Ultrasound Induced Bubble Cavitation and Application to Dissociate Amyloid Fibril. J. Chem. Phys 2016, 145, 174113.

[50] Viet, M. H.; Li, M.; Derreumaux, P.; Nguyen, P. H. Rayleigh-Plesset Equation of the Bubble Stable Cavitation in Water: A Nonequilibrium All-Atom Molecular Dynamics Simulation Study. J. Chem. Phys. 2018, 148 094505.

[51] Viet, M. H.; Phan, M. T.; Li, M.; Derreumaux, P.; Junmei, W.; Van-Oanh, N. T.; Derreumaux, P.; Nguyen, P. H. Molecular mechanism of the cell membrane pore formation induced by bubble stable cavitation. $J$. Phys. Chem. B 2019, 123, 71.

[52] Viet, M. H.; Li, M.; Wang, J.; Derreumaux, P.; Nguyen, P. H. Interaction mechanism between the focused ultrasound and lipid membrane at the molecular level. J. Chem. Phys. 2019, 150, 215101.

[53] Irudayanathan, F. J.; Trasatti, J. P.; Karande, P.; Nangia, S. Molecular Architecture of the Blood Brain Barrier Tight Junction Proteins-A Synergistic Computational and In Vitro Approach. J. Phys. Chem. B 2016, 120, $77-88$.

[54] Irudayanathan, F. J.; Wang, N.; Wang, X.; Nangia, S. Architecture of the paracellular channels formed by claudins of the blood-brain barrier tight junctions. Ann. N. Y. Acad. Sci. 2017, 1405, 131-146.

[55] Rajagopal, N.; Irudayanathan, F. J.; Nangia, S. Computational Nanoscopy of Tight Junctions at the Blood-Brain Barrier Interface. Int J Mol Sci. 2019, 20, 5583.

[56] Piontek, J.; Winkler, L.; Wolburg, H.; Muller, S. L.; Zuleger, N.; Piehl, C.; Wiesner, B.; Krause, G.; Blasig, I. E. Formation of tight junction: determinants of homophilic interaction between classic claudins. The FASEB Journal 2008, 22, 146

[57] Nitta, T.; Hata, M.; Gotoh, S.; Seo, Y.; Sasaki, H. Hashimoto, N.; Furuse, M.; Tsukita, S. Size-selective loosening of the blood-brain barrier in claudin-5-deficient mice. J. Cell Biol. 2003, 161, 653.

[58] Rossa, J.; Lorenz, D.; Ringling, M.; Veshnyakova, A.; Piontek, J. Overexpression of claudin-5 but not claudin-3 induces formation of trans-interaction-dependent multilamellar bodies. Ann N Y Acad Sci. 2012, 125\%, 59-66.

[59] Cording, J.; Kading, J. B. J. N.; Bellmann, C.;
Tscheik, C.; Westphal, J. K.; Milatz, S.; Gunzel, D.; Wolburg, H.; Piontek, J.; et al., In tight junctions, claudins regulate the interactions between occludin, tricellulin and marvelD3, which, inversely, modulate claudin oligomerization. J Cell Sci 2013, 126, 554.

[60] MacKerell, Jr., A. D. et al. All-atom empirical potential for molecular modeling and dynamics studies of proteins. J. Phys. Chem. B 1998, 102, 3586-3616.

[61] Jorgensen, W. L.; Chandrasekhar, J.; Madura, J. D.; Impey, R. W.; Klein, M. L. Comparison of simple potential functions for simulating liquid water. J. Chem. Phys. 1983, 779, 926-935

[62] Lindahl, E.; Hess, B.; van der Spoel, D. GROMACS 3.0: A Package for Molecular Simulation and Trajectory Analysis. J. Mol. Mod. 2001, 7, 306-317.

[63] Berendsen, H. J. C.; Postma, J. P. M.; van Gunsteren, W. F.; Dinola, A.; Haak, J. R. MolecularDynamics With Coupling to an External Bath. J. Chem. Phys. 1984, 81, 3684-3690.

[64] Darden, T.; York, D.; Pedersen, L. Particle Mesh Ewald An $N \cdot \log (\mathrm{N})$ Method for Ewald Sums in Large Systems. J. Chem. Phys. 1993, 98, 10089-10092.

[65] Frishman, D.; Argos, P. Knowledge-Based Protein Secondary Structure Assignment Proteins. Proteins 1995 23, 566-579.

[66] Aryal, M.; Arvanitis, C. D.; Alexander, P. M.; McDannold, N. Ultrasound-mediated blood-brain barrier disruption for targeted drug delivery in the central nervous system. Adv. Drug. Deliv. Rev. 2014, 72, 94-109.

[67] Cheng, K. T.; Wei, K. C.; Liu, H. L. Theranostic Strategy of Focused Ultrasound Induced Blood-Brain Barrier Opening for CNS Disease Treatment. Front. Pharmacol. 2019, 7, 86.

[68] Canney, M. S.; Bailey, M. R.; Crum, L. A. Acoustic characterization of high intensity focused ultrasound fields: A combined measurement and modeling approach. $J$ Acoust Soc Am. 2008, 124, 2406-2420.

[69] Huang, S. L.; MacDonald, R. C. Acoustically active liposomes for drug encapsulation and ultrasound-triggered release. Biochim. Biophys. Acta 2004, 1665, 134-141.

[70] Schroeder, A.; Kost, J.; Barenholz, Y. Ultrasound, liposomes, and drug delivery: Principles for using ultrasound to control the release of drugs from liposomes. Chem. Phys. Lipids 2009, 162, 1-16.

[71] Shinoda, W. Permeability across lipid membranes. Biochimica et Biophysica Acta-Biomembranes 2016, 1858, 2254-2265.

[72] Orsi, M.; Sanderson, W. E.; Essex, J. W. Permeability of Small Molecules through a Lipid Bilayer: A Multiscale Simulation Study. J. Phys. Chem. B 2009, 113, 12019 12029 .

[73] Xiang, T. X.; Anderson, B. D. Molecular dissociation processes in lipid bilayers: a molecular dynamics simulation. J; Chem. Phys. 1999, 110, 1807-1818.

[74] Wang, Y.; Cohen, J.; Boron, W. F.; Schulten, K.; Tajkhorshid, E. Expoloring gas permeability of cellular membranes and membrane channels with molecular dynamics. J. Struct. Biol. 2007, 15\%, 534-544.

[75] Sheikov, N.; McDannold, N.; Jolesz, F.; Zhang, Y. Z.; Tam, K.; Hynynen, K. Brain arterioles show more active vesicular transport of blood-borne tracer molecules than capillaries and venules after focused ultrasound-evoked opening of the blood-brain barrier. Ultrasound Med Biol. 2006, 32, 1399-409. 
[76] Nasica-Labouze, J. et al. Amyloid beta-protein and Alzheimer's disease: when computer simulations complement experimental studies. Chem. Rev. 2015, 115, 3518.

[77] Doig, A. J.; Derreumaux, P. Inhibition of protein aggre- gation and amyloid formation by small molecules. Curr Opin. Struct. Biol. 2015, 30, 50-56. 


\section{0 degree}
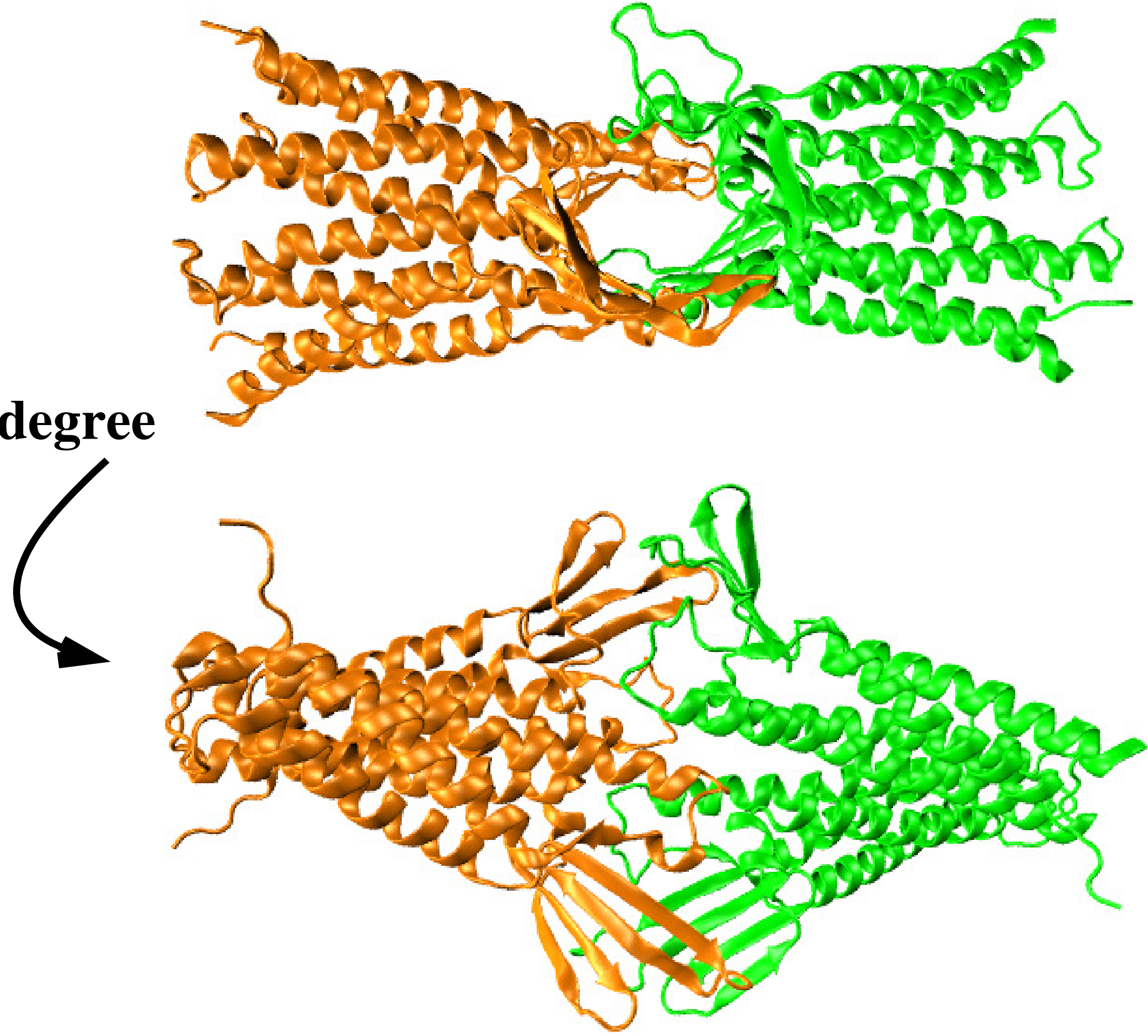

$7_{i}$

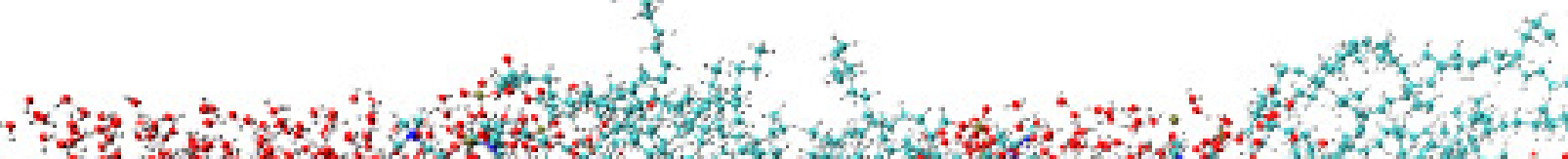

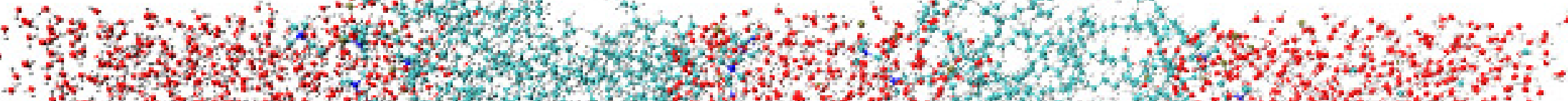

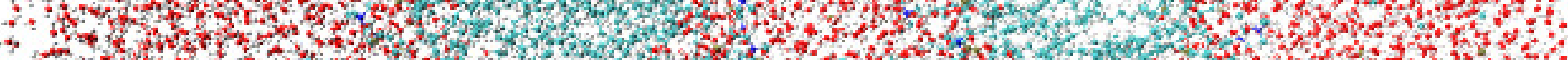

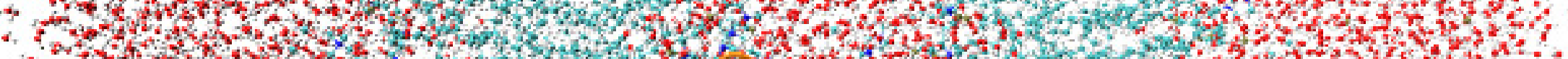

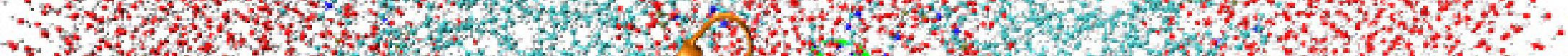

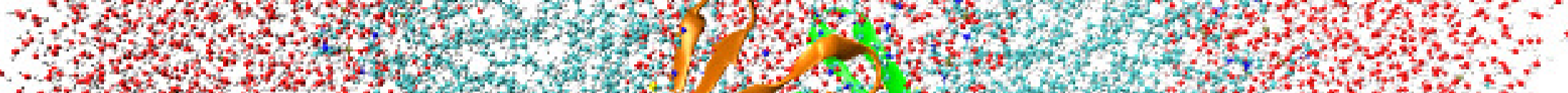

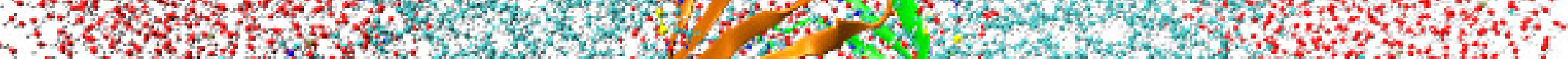

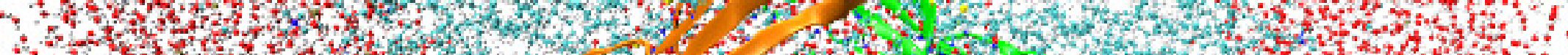

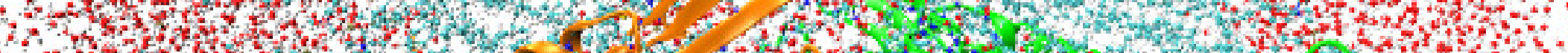

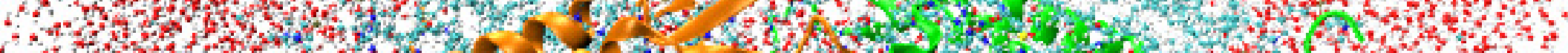

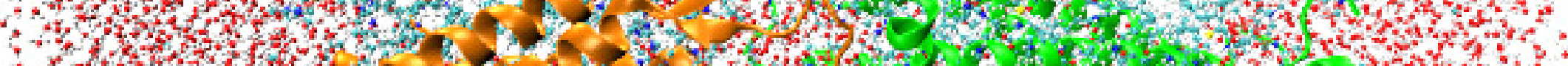

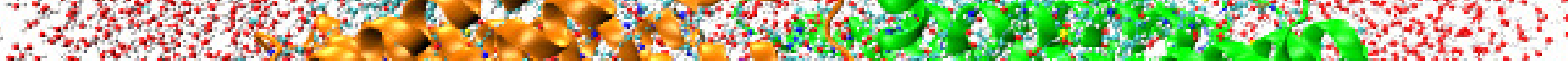

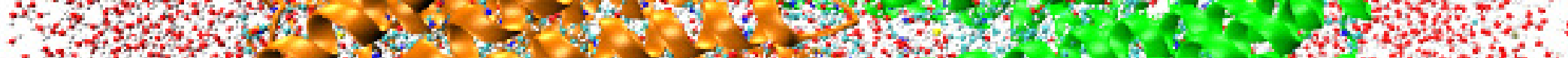

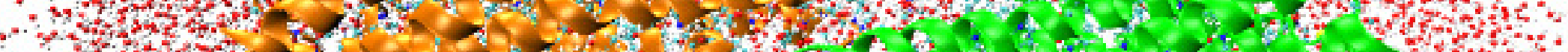

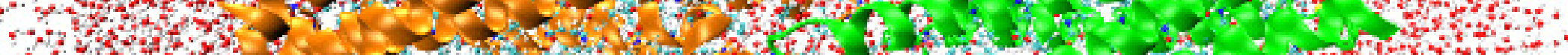

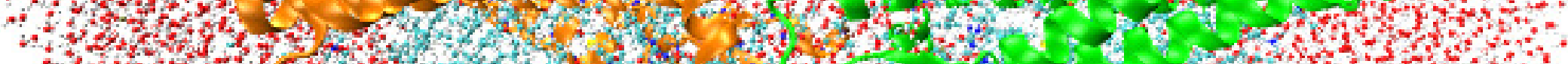

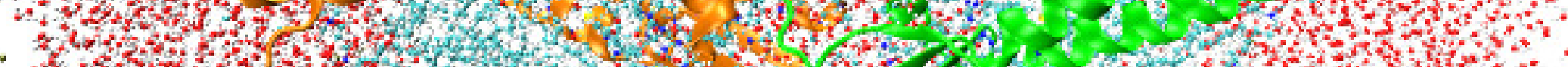

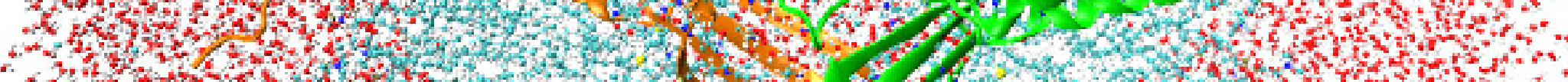

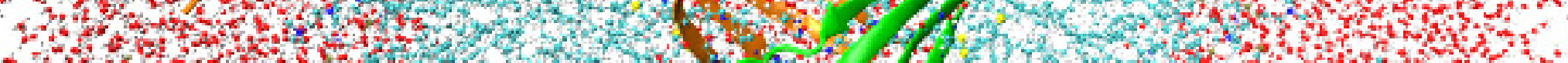

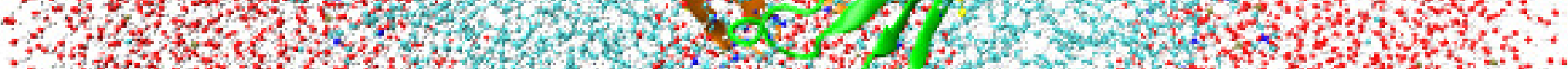

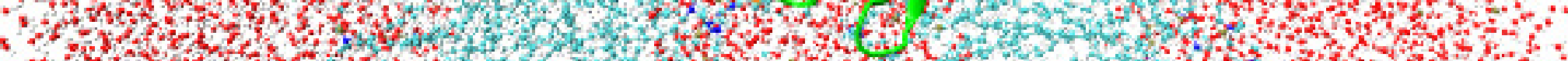

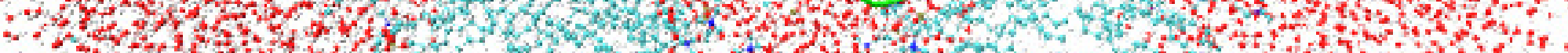

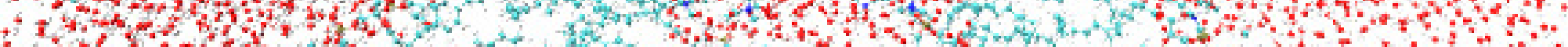

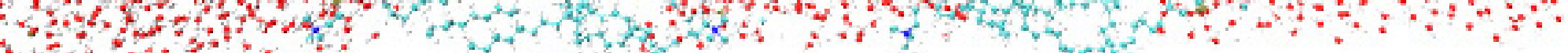




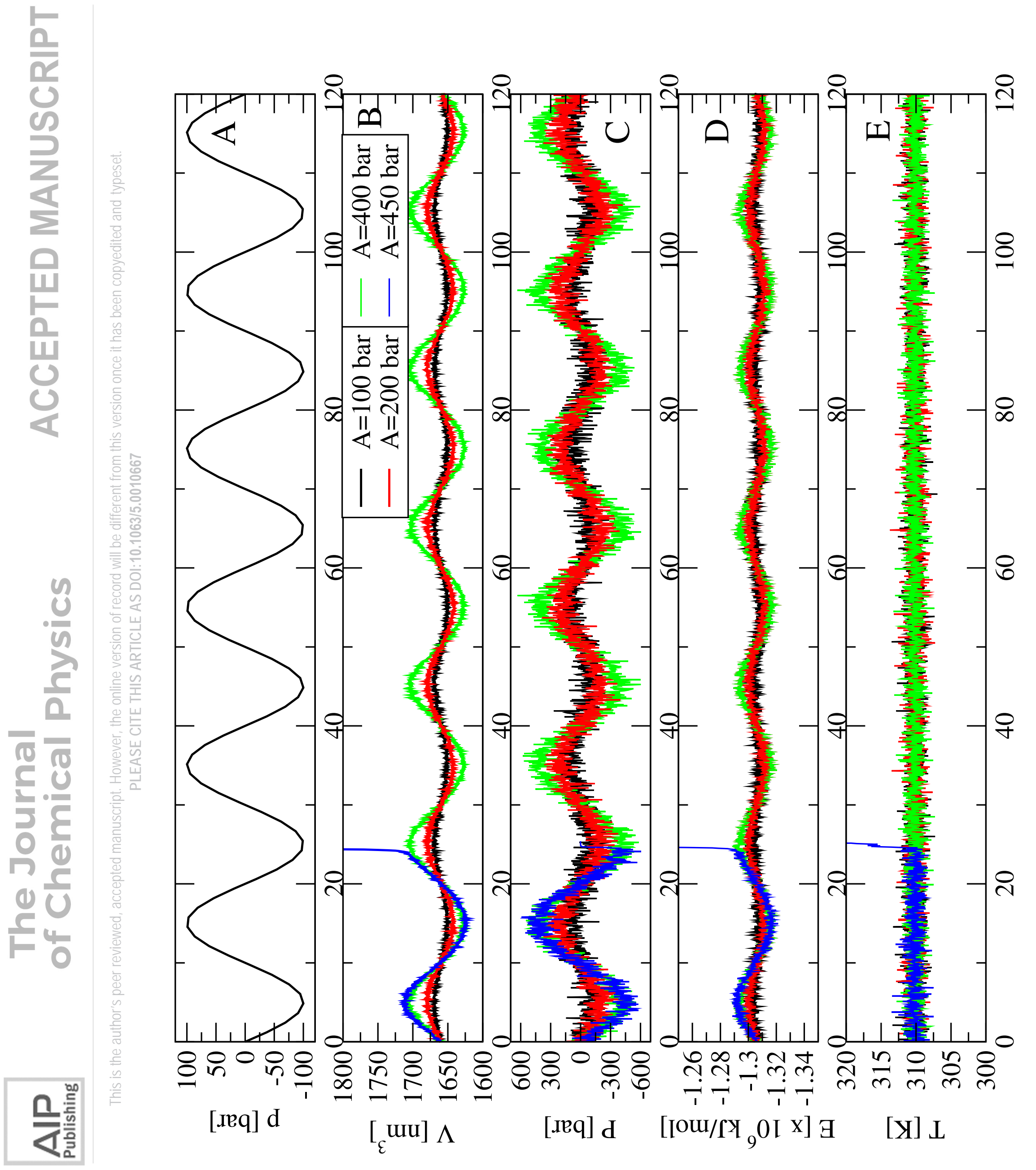




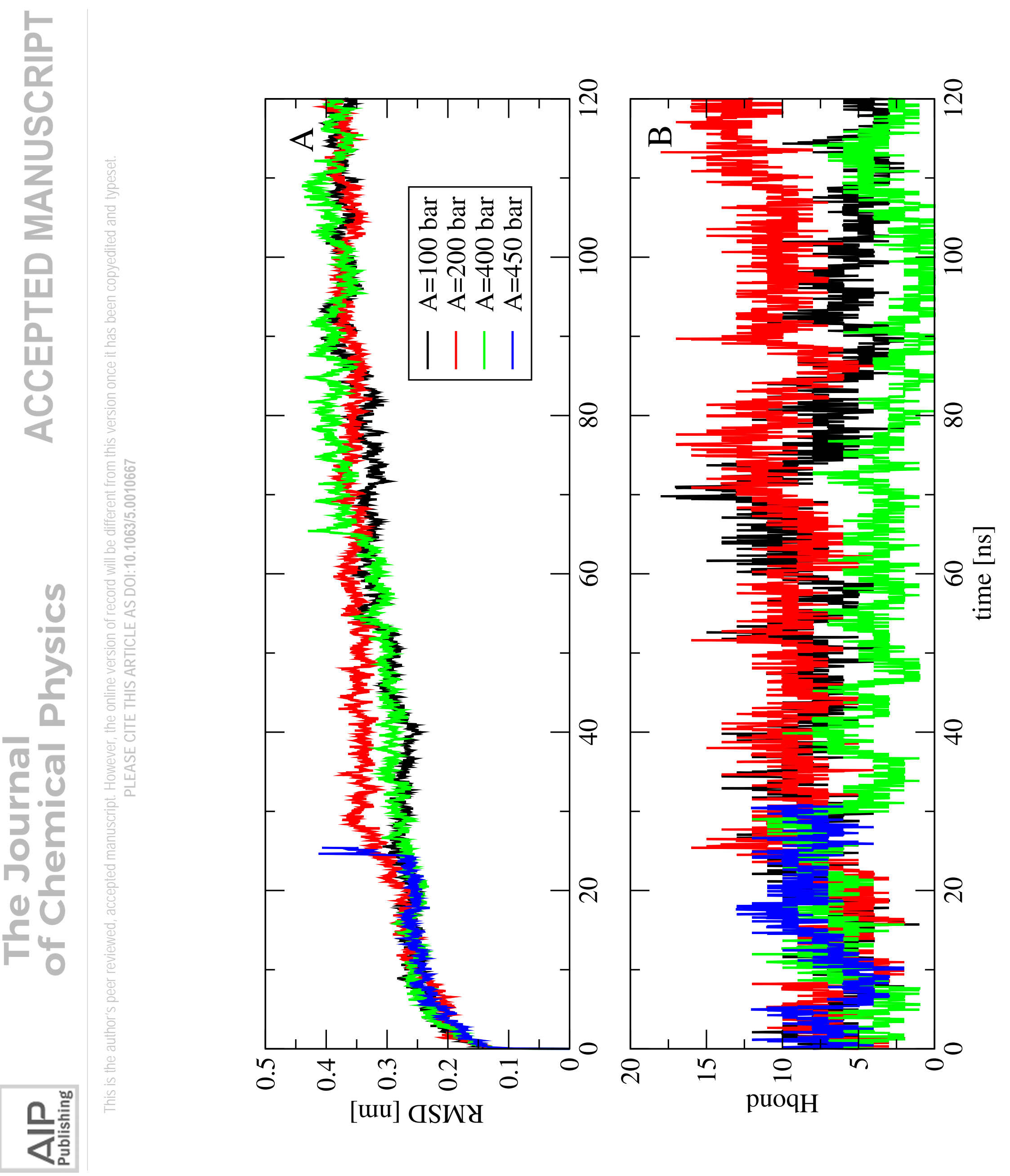



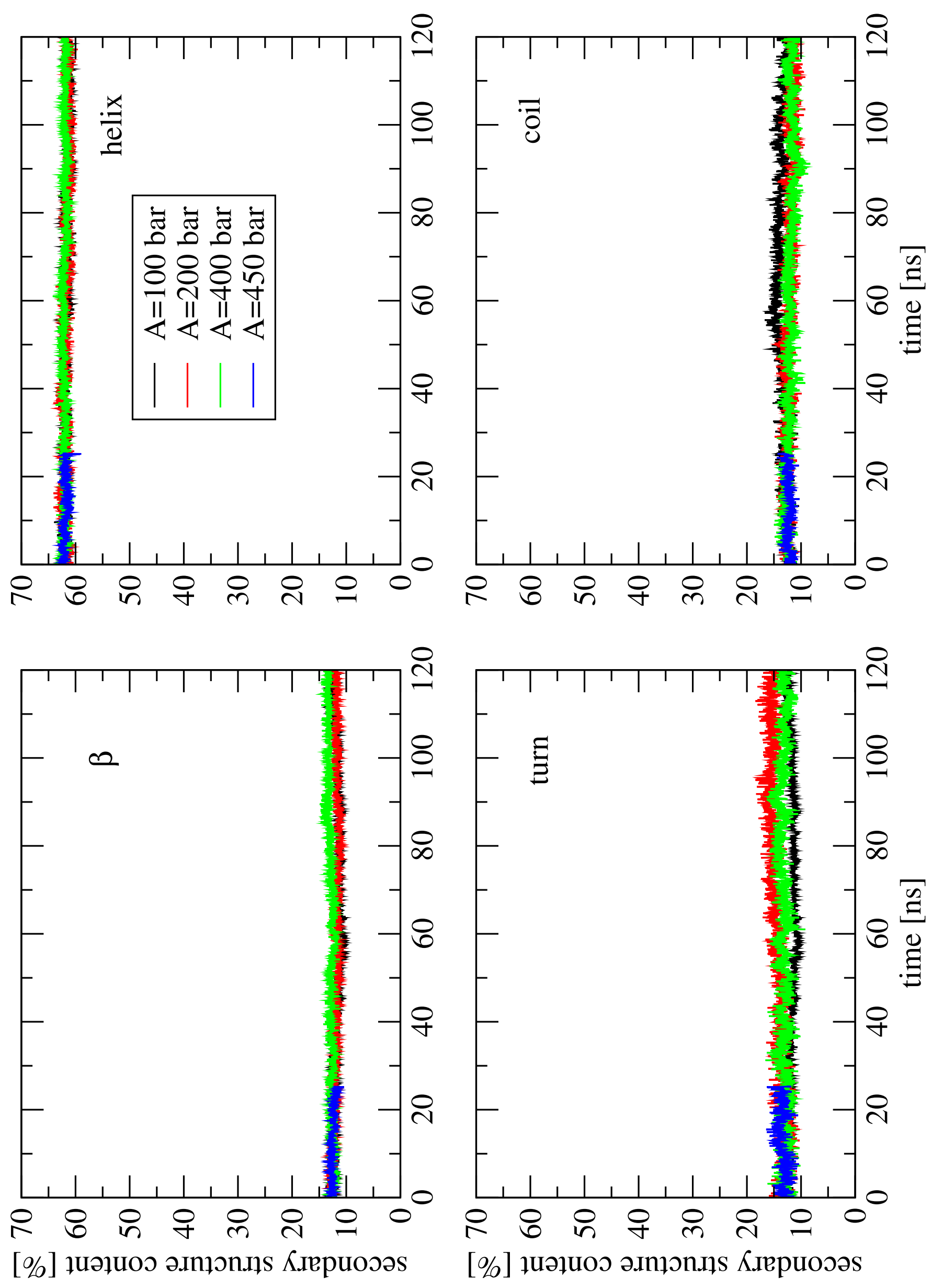


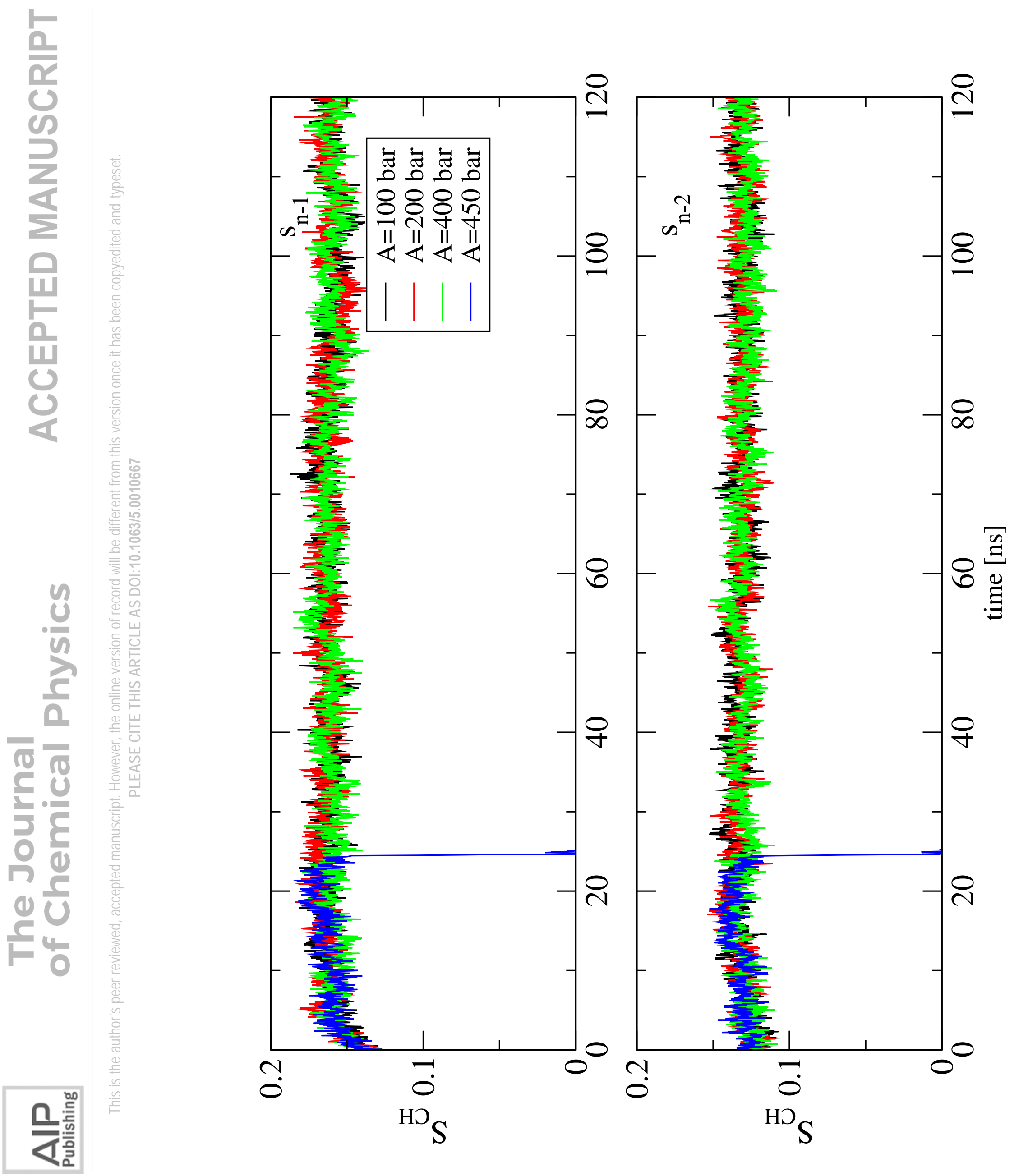




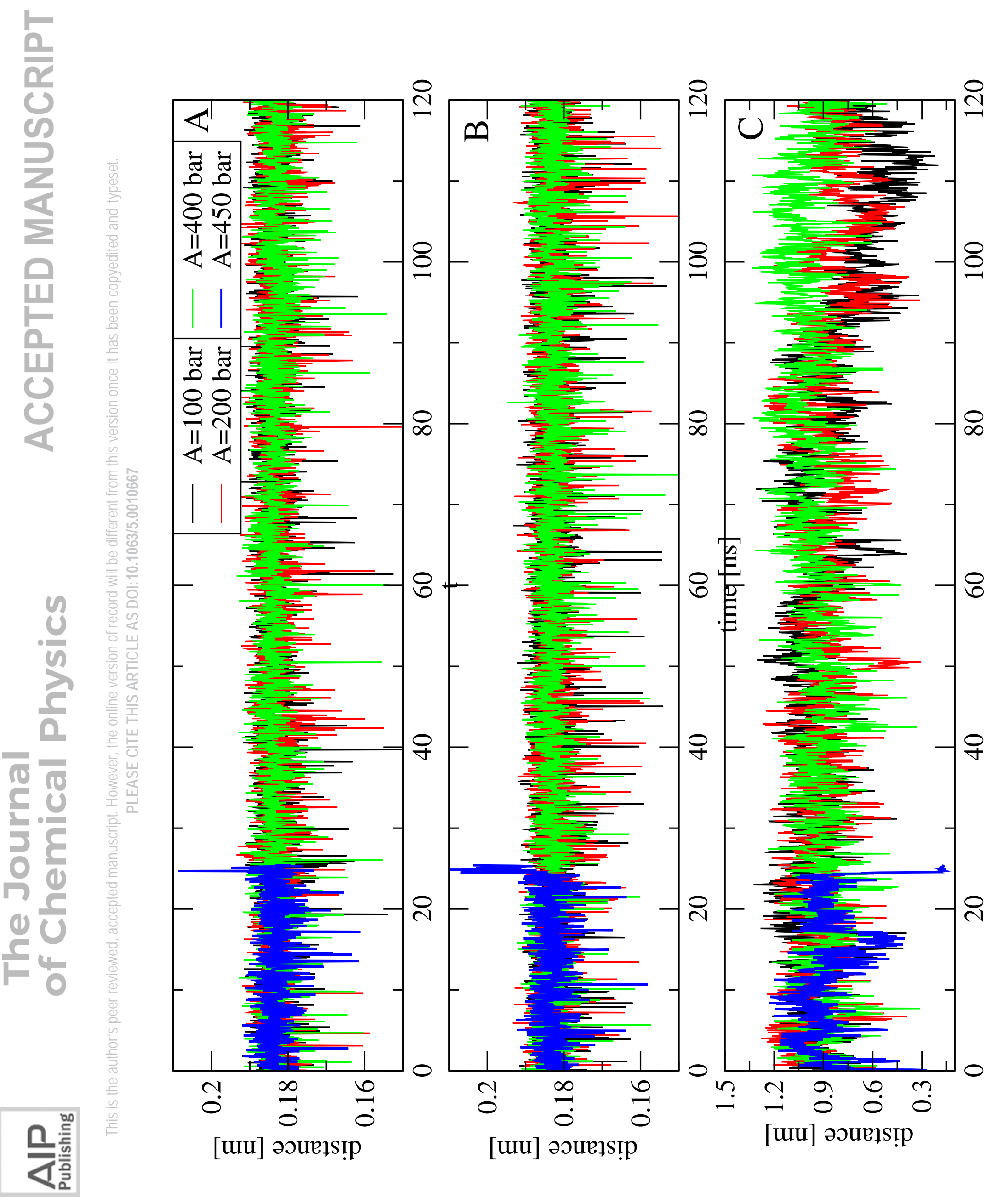


$3+4+\infty+2)$

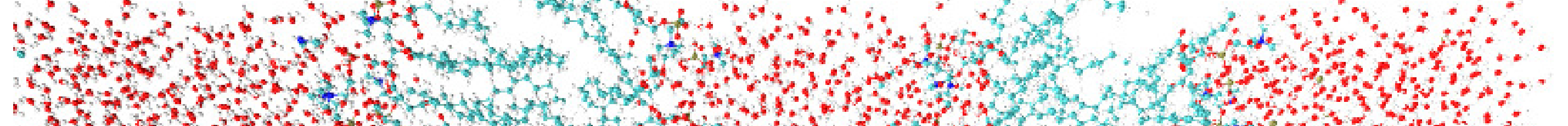

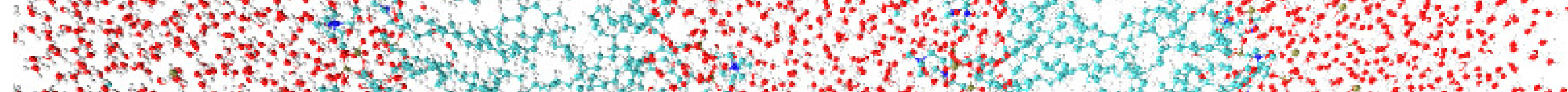

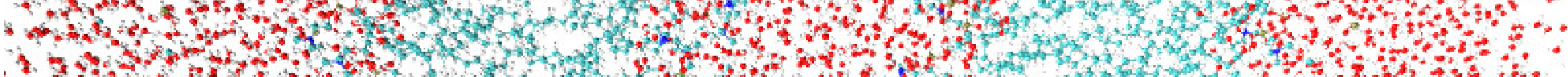

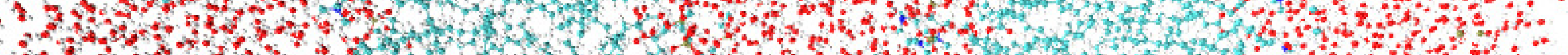

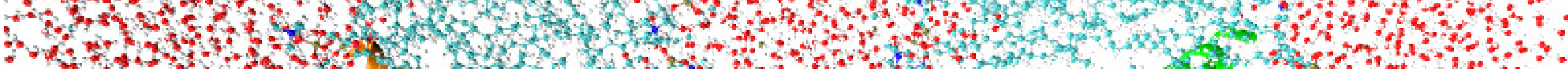

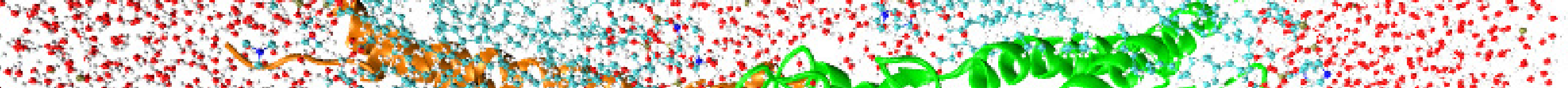

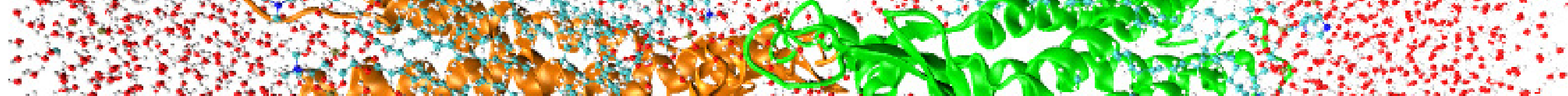

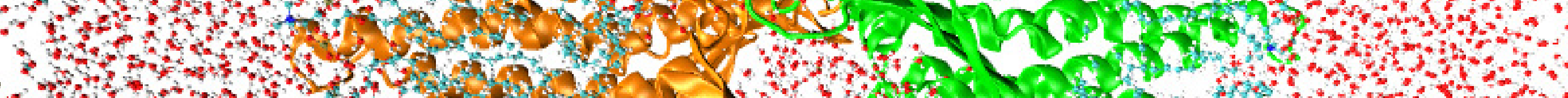

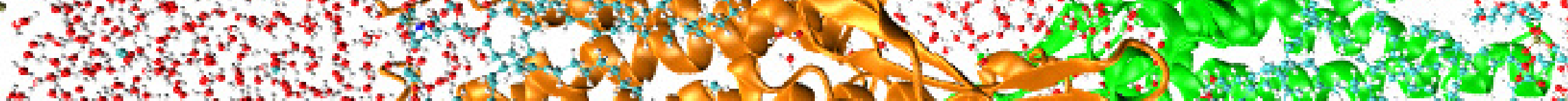

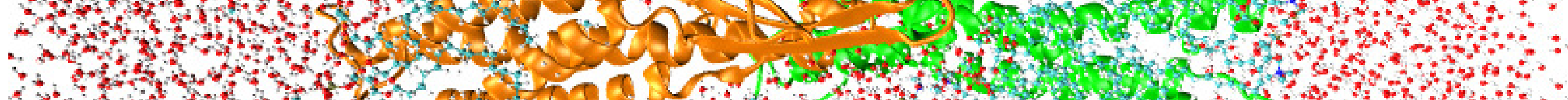

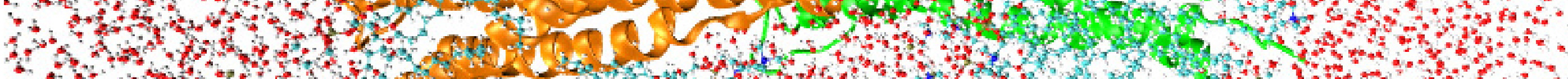
.

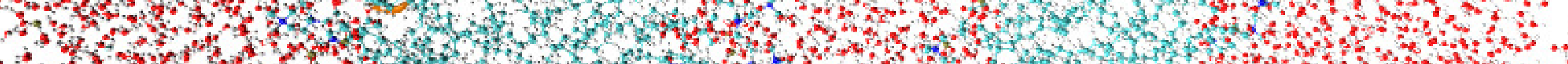

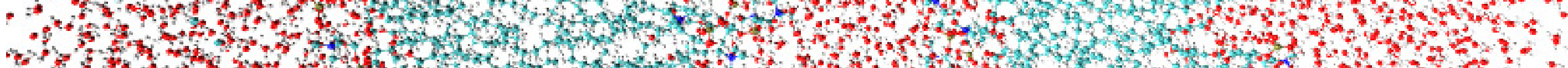

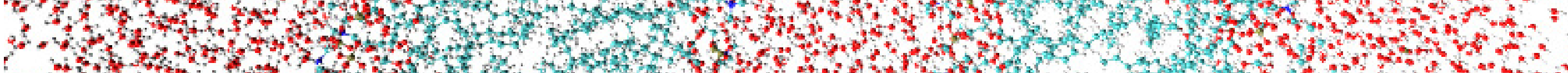

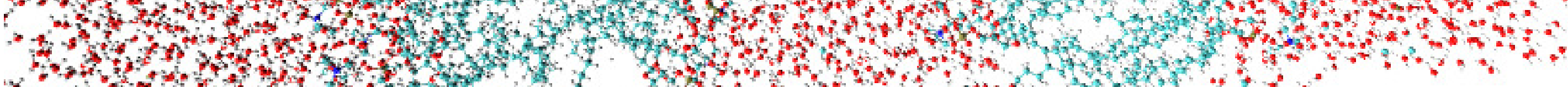
n.t.

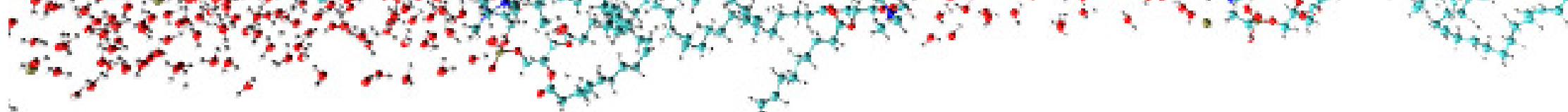

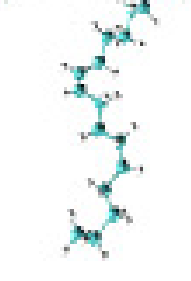

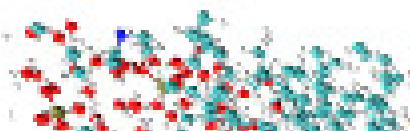

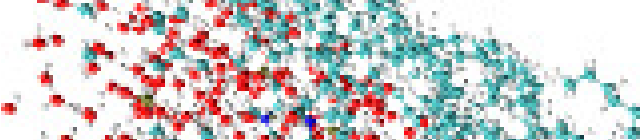

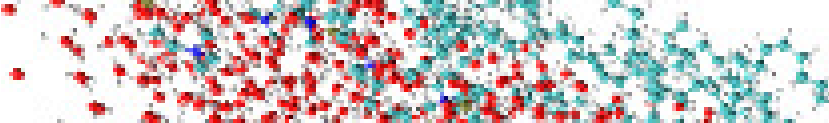

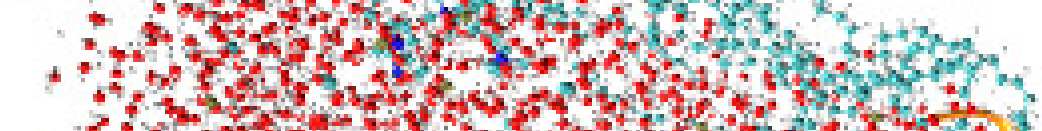

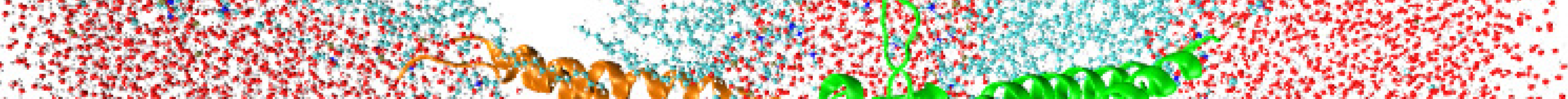

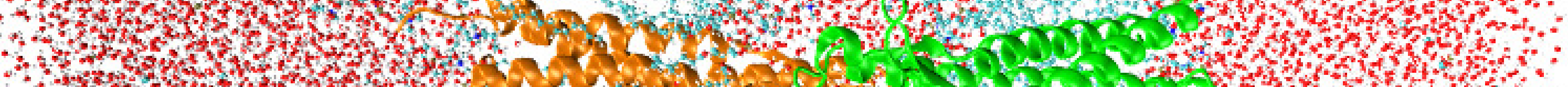

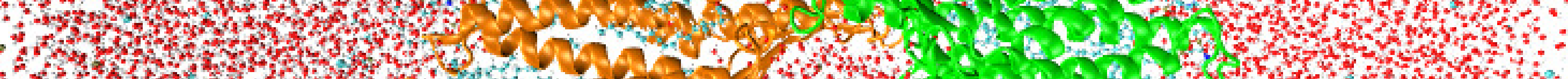

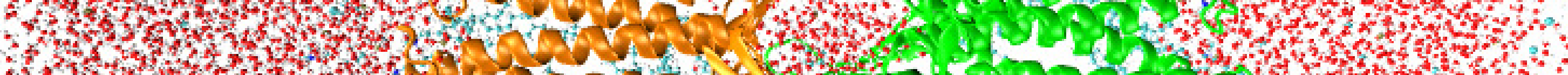

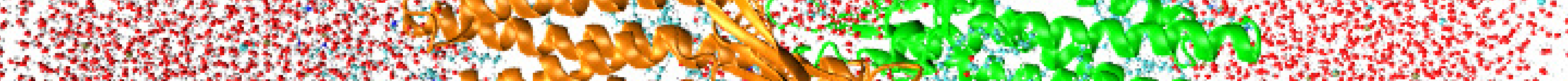

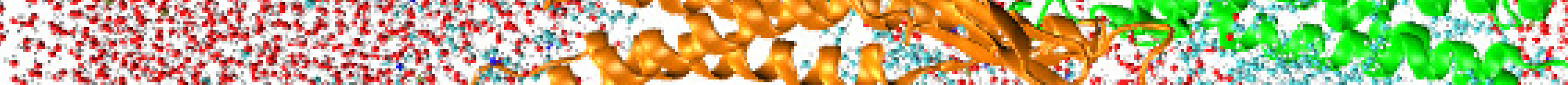

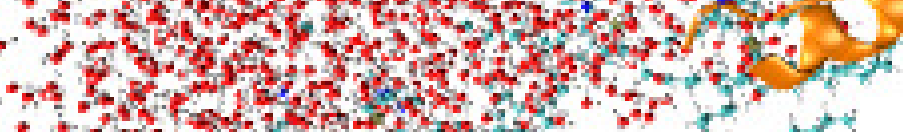

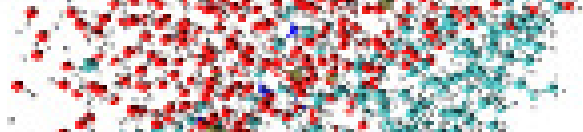

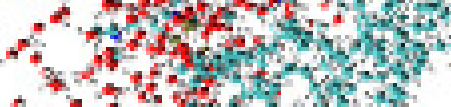
3.t. thentwo

whond $\begin{array}{lll} & & \\ \end{array}$ How W

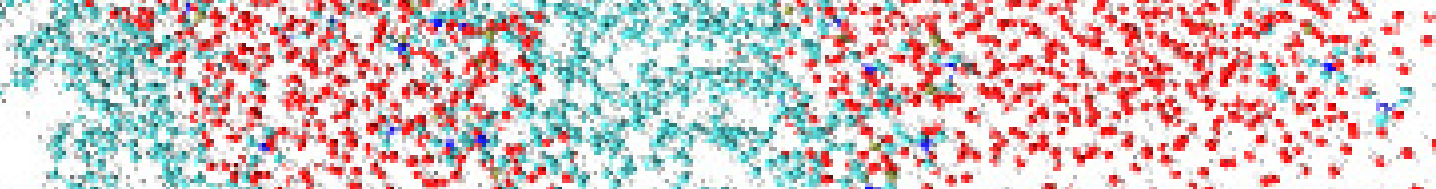

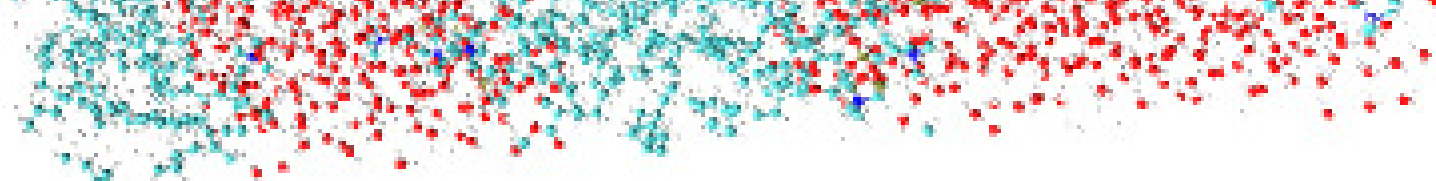

$x^{2}+x^{2}+x^{2}$ (2)

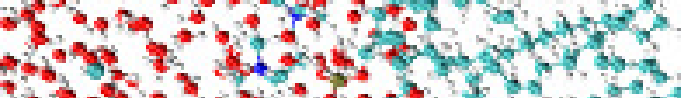

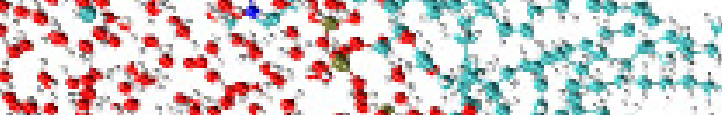

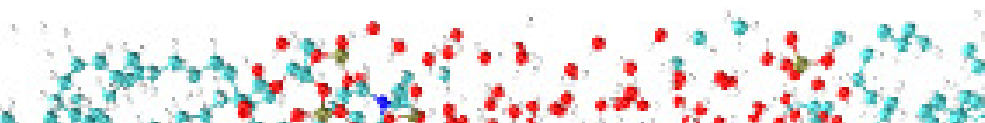

0 ,

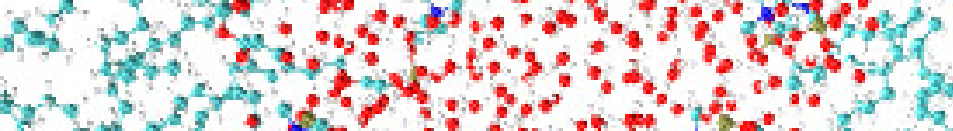

Noth of

6.

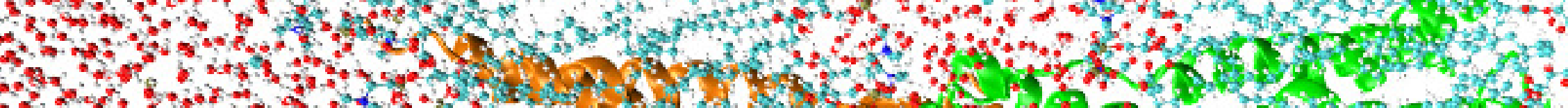

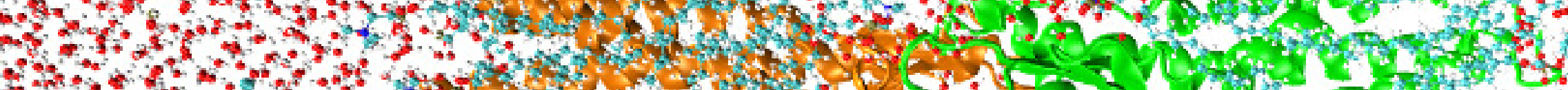

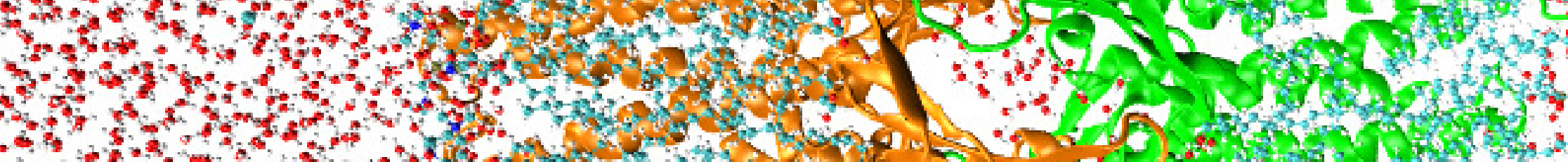

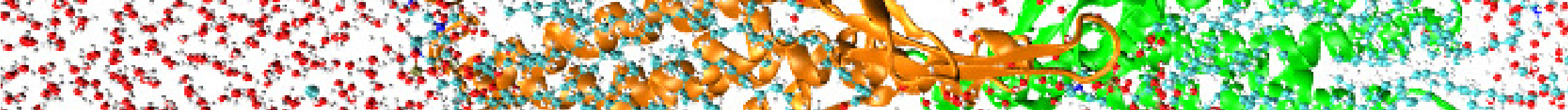

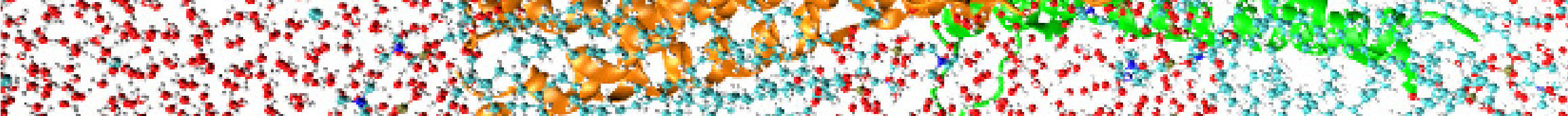

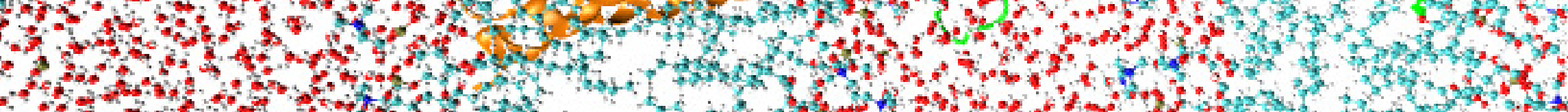

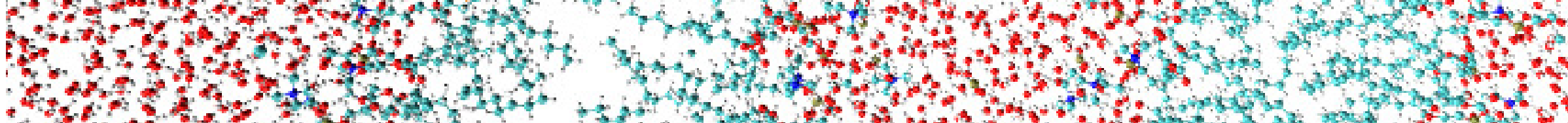
son.t.

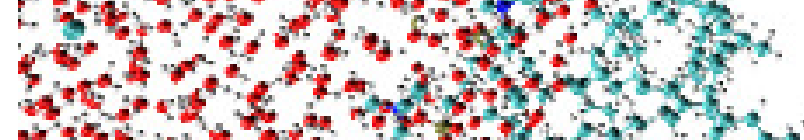

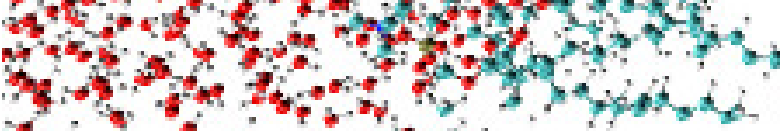

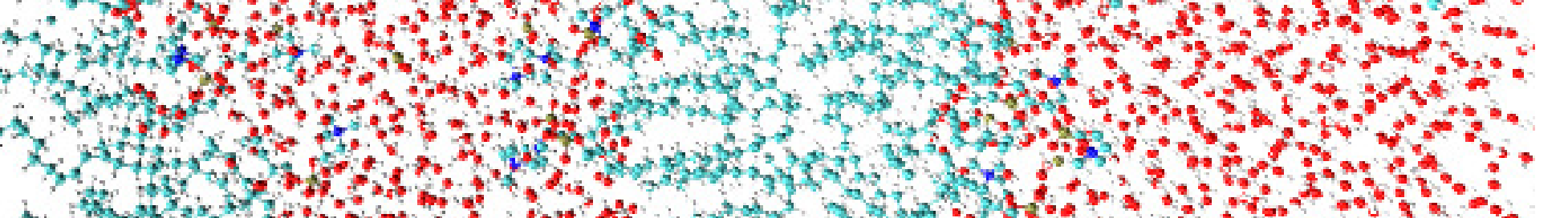
how

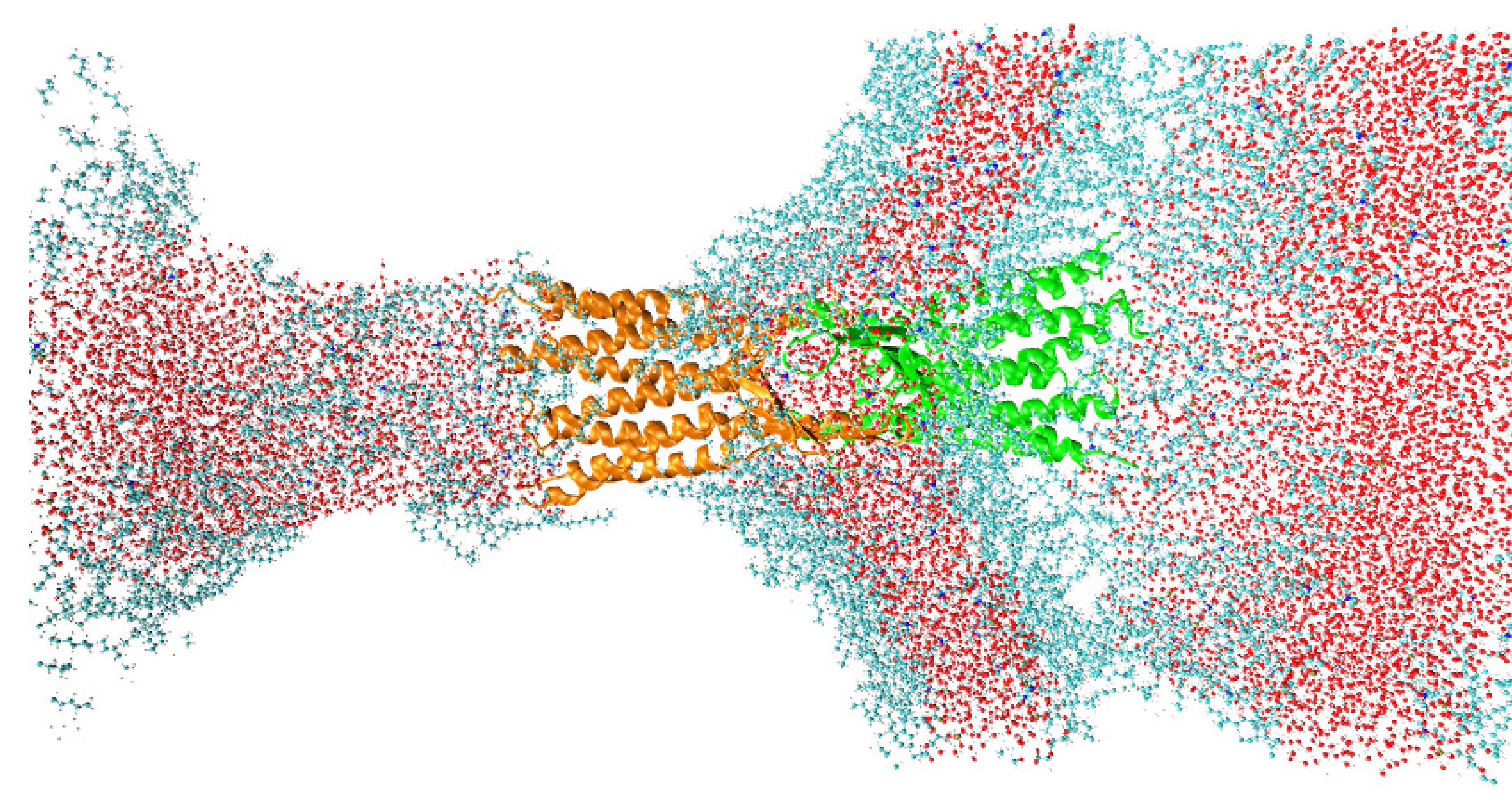

D 

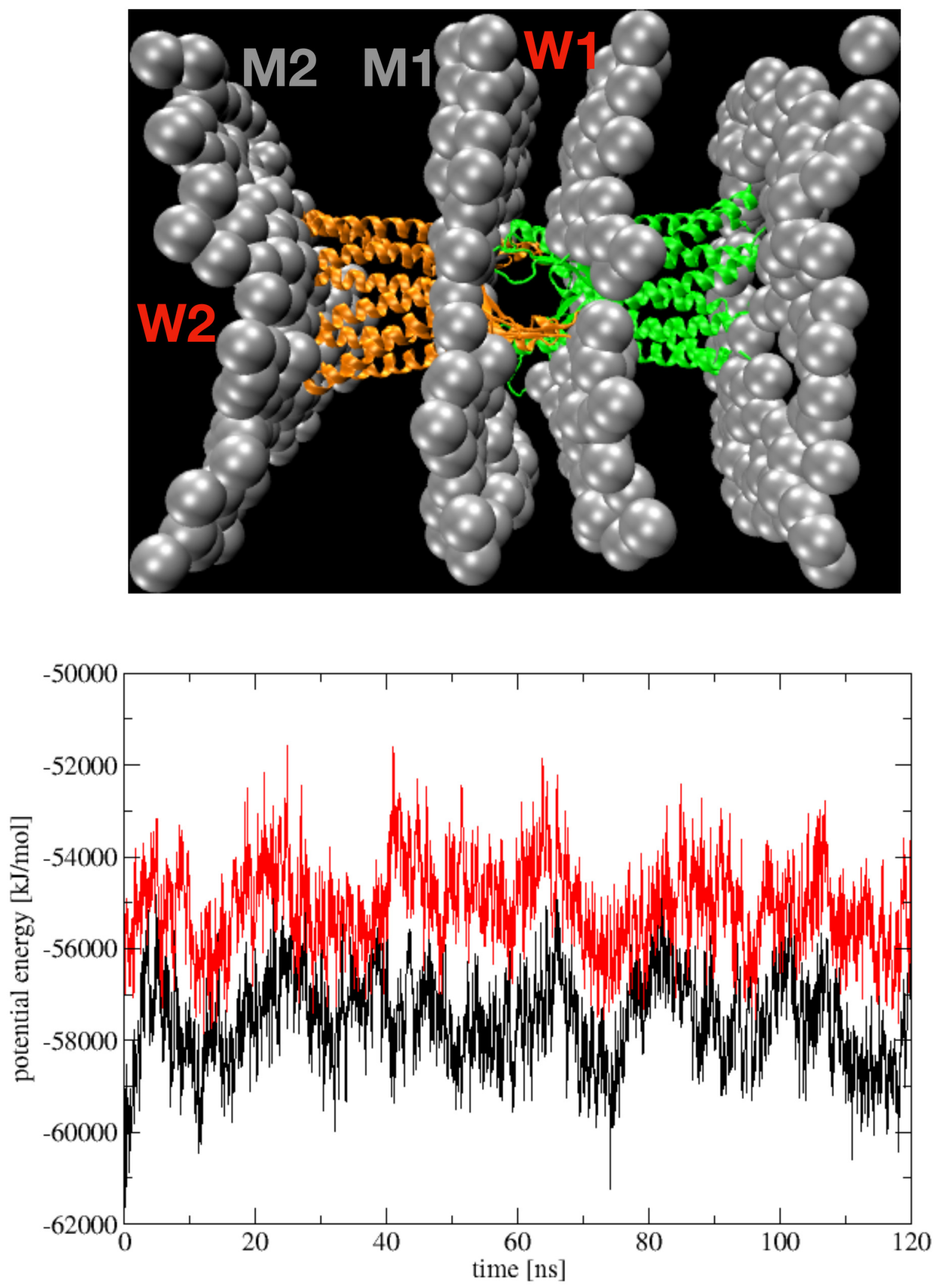


\section{0 bar}
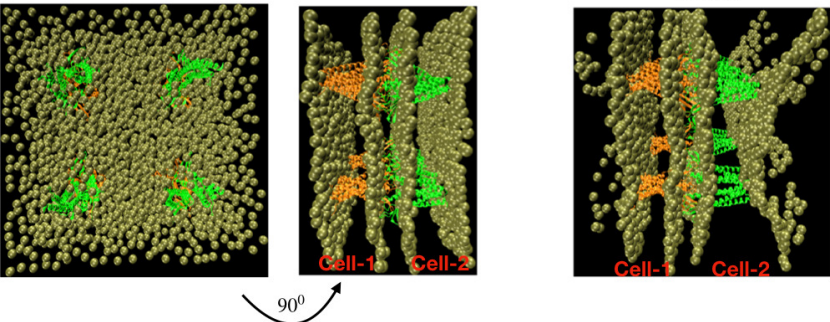